\title{
Chapter 6 \\ Chondrules: Ubiquitous Chondritic Solids Tracking the Evolution of the Solar Protoplanetary Disk
}

\author{
Martin Bizzarro, James N. Connelly, and Alexander N. Krot
}

\begin{abstract}
Chondrite meteorites are samples of primitive asteroidal bodies that have escaped melting and differentiation. The only record of our Solar System's formative stages comes from the earliest solids preserved in chondrites, namely millimetre- to centimetre-sized calcium-aluminium-rich inclusions (CAIs) and chondrules. These solids formed by transient heating events during the lifetime of the solar protoplanetary disk. Collectively, CAIs and chondrules provide timesequenced samples allowing us to probe the composition of the disk material that accreted to form planetesimals and planets. Here, we showcase the current stateof-the-art data with respect to the chronology and stable isotopic compositions of individual chondrules from various chondrite groups and discuss how these data can be used to provide novel insights into the thermal and chemical evolution of the solar protoplanetary disk, including mass transport processes.
\end{abstract}

\subsection{Introduction}

Protoplanetary disks are flattened, rotating structures consisting of cool dust and gas surrounding most young low-mass stars, and are a consequence of the requirement to conserve angular momentum during the gravitational collapse of a prestellar core. Astronomical observations suggest that these disks may only exist for a few million years (Evans et al. 2009), a timescale that corresponds to the period where observable mass accretion to the central star occurs (Williams and Cieza 2011).

\footnotetext{
M. Bizzarro $(\bowtie) \cdot J . N$. Connelly

Centre for Star and Planet Formation, Natural History Museum of Denmark, University

of Copenhagen, Copenhagen, Denmark

e-mail: bizzarro@snm.ku.dk; connelly@snm.ku.dk
}

\section{A.N. Krot}

Hawai'i Institute of Geophysics and Planetology, School of Ocean and Earth Science and Technology, University of Hawaii at Mānoa, Honolulu, HI, USA

e-mail: sasha@higp.hawaii.edu 
Some material, however, coalesces into centimetre-sized particles that accrete to form larger asteroidal bodies, which represent the building blocks of planetary systems. Thus, the study of these accreting protoplanetary disks provides direct insights into the initial conditions for planet formation.

In the Solar System, a record of the earliest evolutionary stages of the protoplanetary disk is preserved in chondritic meteorites (chondrites), which are fragments of asteroids that avoided melting and differentiation. Based on the bulk chemical and isotopic compositions, mineralogy and petrography, 15 chondrite groups and grouplets comprise three major chondrite classes, carbonaceous $(\mathrm{CI}, \mathrm{CM}, \mathrm{CR}, \mathrm{CV}$, $\mathrm{CK}, \mathrm{CO}, \mathrm{CB}, \mathrm{CH})$, ordinary (H, L, LL), enstatite (EH, EL), as well as K and R, are currently recognised. Most chondrites consist of chondrules, refractory inclusions [Ca,Al-rich inclusions (CAIs) and amoeboid olivine aggregates (AOAs)], and finegrained matrix (Fig. 6.1a-d). The only exception are CB carbonaceous chondrites, which lack fine-grained matrix and contain anomalously high abundance of $\mathrm{Fe}, \mathrm{Ni}$ metal (up to 70 vol\%; Fig. 6.1e, f).

In primitive (unmetamorphosed and unaltered) chondrites, fine-grained matrices are complex mixtures of micrometre-sized crystalline magnesian olivine and low-Ca pyroxene, amorphous ferromagnesian silicates, Fe,Ni-metal, sulfides, and organics (Greshake 1997; Abreu and Brearley 2010). Although primitive chondrite matrices are typically considered as volatile-rich thermally unprocessed materials, a significant fraction of this material was probably vaporised and recondensed during high-temperature transient heating events associated with formation of chondrules and refractory inclusions (Scott and Krot 2005).

CAIs represent the oldest Solar System dated solids and, thus, define its age at $4567.3 \pm 0.16 \mathrm{Myr}$ (Connelly et al. 2012). It is commonly accepted that CAIs formed in a hot (ambient temperature above $\sim 1300 \mathrm{~K}$ ) disk region characterised by approximately solar oxygen isotopic composition near the proto-Sun by evaporation, condensation and aggregation processes during a brief time interval that corresponded to high stellar mass accretion rates $\left(\sim 10^{-5} \mathrm{M}_{\odot}\right.$ year $\left.^{-1}\right)$ (Krot et al. 2009). Formation of CAIs near the proto-Sun is also indicated by the presence in these objects of the short-lived radioisotope ${ }^{10} \mathrm{Be}$ (half-life of $\sim 1.4 \mathrm{Myr}$ ) formed by solar energetic particle irradiation (McKeegan et al. 2000). Some CAIs were subsequently melted, most in the same disk region. Following their formation, CAIs were transported to large radial distances where they accreted into chondritic and cometary parent bodies. Most chondrules formed by melting (typically incomplete) of solid precursor material during transient heating events (peak temperature of $\sim 2000 \mathrm{~K}$ ) of unknown nature in different, relatively cold dust-rich regions throughout the protoplanetary disk during its entire lifetime (Connelly et al. 2012). Therefore, CAIs and chondrules provide time-sequenced samples allowing us to probe the composition of the disk material that accreted to form planetesimals and planets.

Judging by their sheer abundance in chondrites, which in some cases reaches $80 \%$ by volume, chondrules must be the product of one of the most energetic processes that operated in the early Solar System. The majority of chondrules formed as melt droplets in high-density regions of the protoplanetary disk and accumulated 


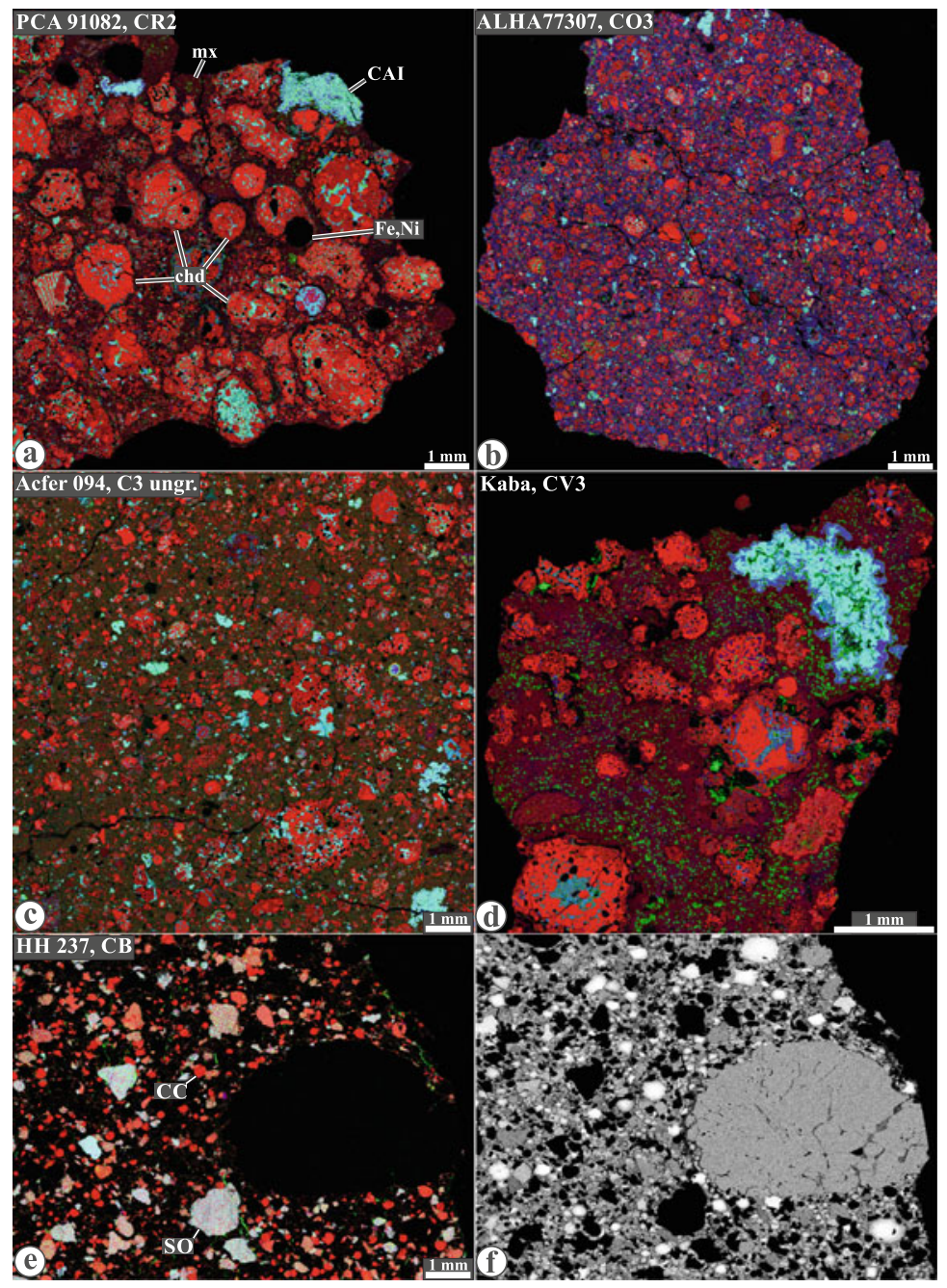

Fig. 6.1 Combined X-ray elemental maps in $\mathrm{Mg}$ (red), $\mathrm{Ca}$ (green) and $\mathrm{Al}$ (blue) of the $\mathrm{CR}$ (Renazzo type), CO (Ornans type), CV (Vigarano type), CB (Bencubbin type) and Acfer 094 (ungrouped) carbonaceous chondrites; (d) X-ray elemental map in $\mathrm{Ni}$ of $\mathrm{HH}$ 237. Typical chondrites consist of chondrules (reddish), Ca,Al-rich inclusions (CAIs, bluish and greenish), and $\mathrm{Fe}, \mathrm{Ni}$-metal (Fe,Ni, black in "(a)-(e)"), all surrounded by fine-grained matrix (mx). There are significant variations in chondrule sizes and chondrule/matrix ratio among the chondrite groups. The majority of chondrules have porphyritic textures (see Fig. 6.2a-d). The CB metal-rich carbonaceous chondrites have no matrix, contain very rare CAIs and anomalously high abundance of $\mathrm{Fe}, \mathrm{Ni}$-metal (up to $70 \mathrm{vol} \%$ ); chondrules have exclusively non-porphyritic, cryptocrystalline (CC) and skeletal olivine (SO) textures (see Fig. 6.2e, f) 


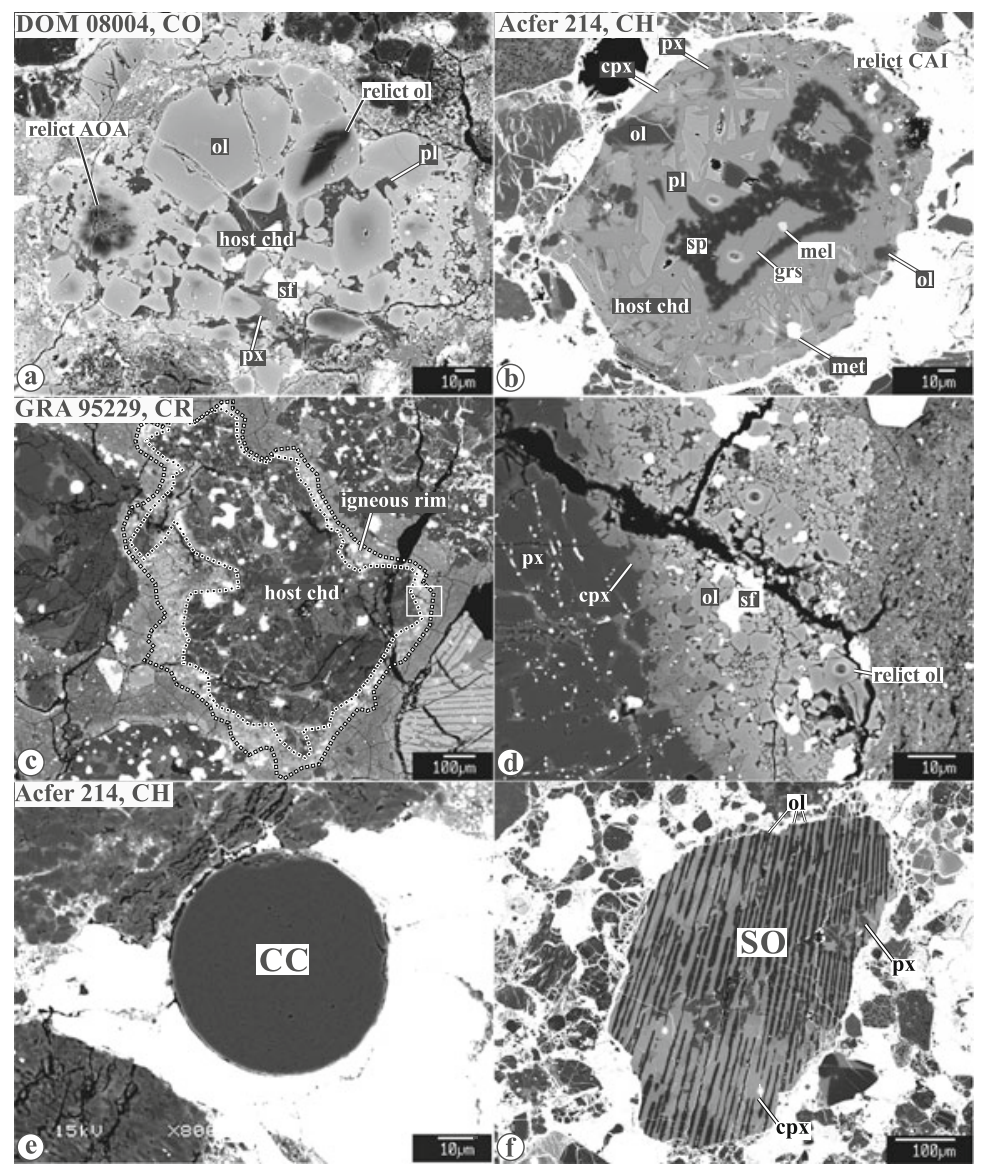

Fig. 6.2 Backscattered electron images of (a-d) porphyritic and (e, f) non-porphyritic chondrules in $\mathrm{CO}, \mathrm{CR}$, and $\mathrm{CH}$ carbonaceous chondrites. The porphyritic chondrules consist of ferromagnesian olivine and pyroxenes, glassy or crystalline mesostasis, Fe,Ni-metal and sulfides. Porphyritic chondrules commonly contain relict grains $(\mathbf{a}, \mathbf{b})$, indicative of incomplete melting of chondrule precursors. The relict grains include fragments of CAIs, AOAs, and chondrules of earlier generations. Some chondrules are surrounded by finer-grained igneous rims, indicative of repeatable melting events experienced by these chondrules $(\mathbf{c}, \mathbf{d})$. The non-porphyritic chondrules contain neither relict grains nor igneous rims, suggesting that they experienced crystallisation from complete melts. $\mathrm{cpx}=$ high-Ca pyroxene; $\mathrm{Fe}, \mathrm{Ni}=\mathrm{Fe}, \mathrm{Ni}-$ metal; grs $=$ grossite; mel = melilite; $\mathrm{ol}=$ olivine; $\mathrm{pl}=$ plagioclase; $\mathrm{px}=$ low-Ca pyroxene; $\mathrm{sf}=\mathrm{Fe}$-sulfide; $\mathrm{sp}=$ spinel

in the disk mid-plane together with other chondritic components. Chondrules are mainly composed of olivine $\left((\mathrm{Fe}, \mathrm{Mg})_{2} \mathrm{SiO}_{4}\right)$ and pyroxene $\left((\mathrm{Fe}, \mathrm{Mg}) \mathrm{SiO}_{3}\right)$ minerals, which crystallised within minutes to days between $\sim 1800$ and $\sim 1300 \mathrm{~K}$ (Scott 2007). Several heat sources have been proposed for the thermal processing of chondrule precursors, including shock waves (Boss and Graham 1993; Connolly and Love 1998; Hood 1998; Connolly et al. 2006), current sheets (Joung et al. 2004), 
X-winds (Shu et al. 1997), magnetised disk wind (Salmeron and Ireland 2012), and colliding planetesimals (Asphaug et al. 2011; Sanders and Scott 2012). A longstanding paradigm used to constrain chondrule-formation models is the so-called chemical complementarity that apparently exists between chondrules and matrix in individual chondrite groups (Hezel and Palme 2010; Palme et al. 2015; Ebel et al. 2016). In this model, it is proposed that chondrules and matrix are genetically related and formed in highly localised regions of the protoplanetary disk. The chronology of chondrule formation is typically based on the short-lived ${ }^{26} \mathrm{Al}$ to ${ }^{26} \mathrm{Mg}$ decay system $\left[{ }^{26} \mathrm{Al}\right.$ decays to ${ }^{26} \mathrm{Mg}$ with a half-life of $0.705 \mathrm{Myr}$ (Norris et al. 1983)]. Assuming that ${ }^{26} \mathrm{Al}$ was uniformly distributed in the protoplanetary disk with the canonical ${ }^{26} \mathrm{Al} /{ }^{27} \mathrm{Al}$ ratio of $\sim 5 \times 10^{-5}$ commonly observed in CAIs, the ${ }^{26} \mathrm{Al}-{ }^{26} \mathrm{Mg}$ systematics of chondrules suggest that these objects formed $>1$ Myr after CAIs and rapidly accreted into chondrite parent bodies together with matrix in discrete events during the lifetime of the disk. In this view, chondrule formation is restricted to the inner regions of the solar protoplanetary disk.

However, a number of recent studies investigating the absolute chronology of chondrule formation as well as the isotopic systematics of individual chondrules from various chondritic meteorites require a reassessment of current thinking with respect to the formation history of chondrules as well as the parent asteroids of chondrite meteorites. For example, the absolute isotopic dates of individual chondrules suggest that the formation of these objects started contemporaneously with the condensation and melting of CAIs and lasted $3.5 \mathrm{Myr}$ (Connelly et al. 2012), which indicate the existence of multiple generations of chondrules within individual chondrites. Moreover, variability in the titanium and chromium stable isotope compositions of chondrules from individual chondrites suggests that these objects or their precursor was formed in distinct regions of the protoplanetary disk and subsequently transported to the accretion regions of their respective parent bodies (Trinquier et al. 2009; Van Kooten et al. 2016; Olsen et al. 2016). These data are at odds with the traditional view of a short formation history for chondrule population from individual chondrites, the basic concept of chondrulematrix complementarity as well as the timescales and style of chondrite parent body accretion. In this contribution, we review the current state-of-the-art data with respect to the chronology and stable isotopic compositions of individual chondrules from various chondrite groups and discuss how these data can be used to provide novel insights into the thermal and chemical evolution of the solar protoplanetary disk, including mass transport processes.

\subsection{Basic Petrological and Chemical Features of Chondrules}

In typical (i.e. not metal-rich) chondrites, most chondrules have porphyritic textures (Figs. 6.1a-d and 6.2a-d) and consist of olivine and/or low-Ca pyroxene phenocrysts surrounded by glassy or microcrystalline mesostasis. Chondrules show large variations in mineral and bulk chemical compositions, allowing us 
to distinguish magnesian $(\mathrm{Fa}=$ atomic ratio $\mathrm{Fe} /(\mathrm{Fe}+\mathrm{Mg}) \times 100$ or $\mathrm{Fs}=$ atomic ratio $\mathrm{Fe} /(\mathrm{Fe}+\mathrm{Mg}+\mathrm{Ca}) \times 100<10 \mathrm{~mol} \%$; type $\mathrm{I})$, ferroan $(\mathrm{Fa}$ or $\mathrm{Fs}>10 \mathrm{~mol} \%$; type II), and aluminum-rich (bulk $\mathrm{Al}_{2} \mathrm{O}_{3}>10 \mathrm{wt} \%$ ) chondrules. Chondrules with non-porphyritic textures (cryptocrystalline and barred/skeletal olivine) are dominant in $\mathrm{CB}$ chondrites, abundant in $\mathrm{CH}$ chondrites (Figs. 6.1e, f and 6.2e, f), but relatively rare in other chondrite groups. Chondrules are on average depleted in siderophile (iron-loving), chalcophile (sulfide-loving) and moderately volatile (having condensation temperature $<1000 \mathrm{~K}$ ) lithophile (silicate-loving) elements compared to whole rock; refractory lithophile elements $(\mathrm{Ca}, \mathrm{Al}, \mathrm{Ti}$, and rare earth elements (REEs)] are approximately solar (Grossman et al. 1988).

On a typical three-isotope oxygen diagram, $\delta^{17} \mathrm{O}$ vs. $\delta^{18} \mathrm{O}\left[\delta^{i} \mathrm{O}=\left({ }^{i} \mathrm{O} /{ }^{16} \mathrm{O}_{\text {sample }}\right.\right.$ $\left./{ }^{i} \mathrm{O} /{ }^{16} \mathrm{O}_{\text {SMOW }}-1\right) \times 1000, i=17$ or 18 ], chondrules generally plot along a slope $\sim 1.0$ mass-independent fractionation line within $\pm 5 \%$ of the terrestrial fractionation line (see Fig. 6.3). In unmetamorphosed chondrites, olivine and lowCa pyroxene phenocrysts and mesostasis in an individual chondrule have similar oxygen-isotope compositions (Tenner et al. 2015). Porphyritic chondrules are thought to have formed by incomplete melting of solid precursors resulting in

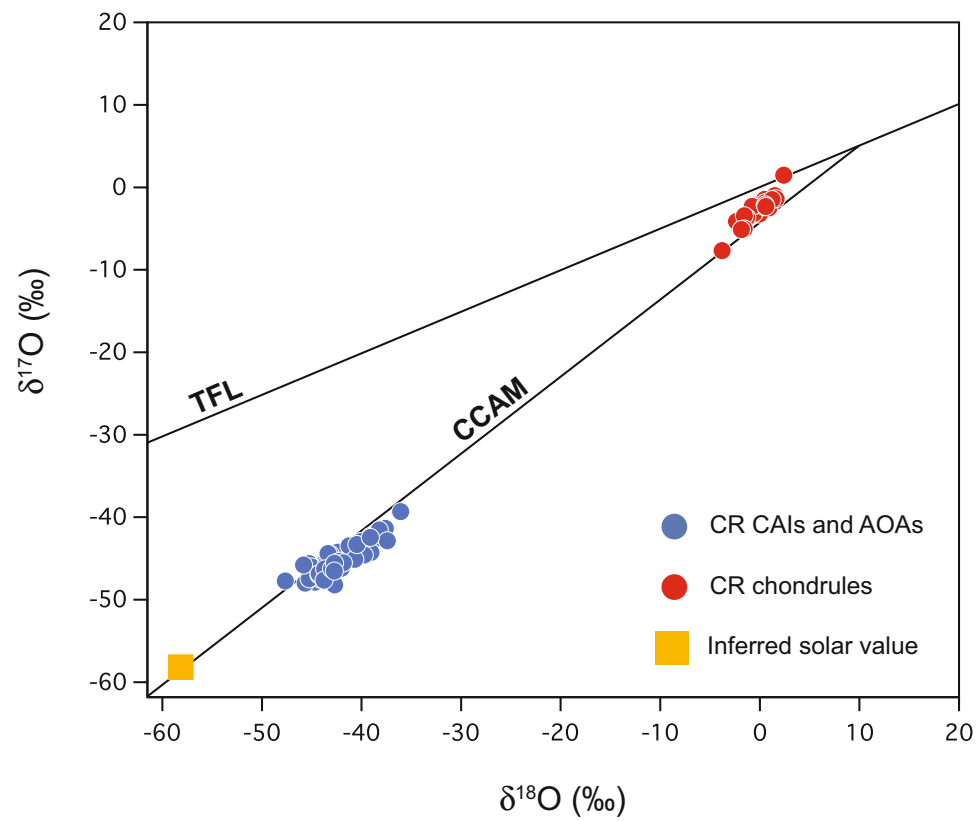

Fig. 6.3 Oxygen-isotope compositions of CAIs and amoeboid olivine aggregates (AOA) and chondrules from CR carbonaceous chondrites. The refractory inclusions and chondrules are isotopically uniform, but have different oxygen-isotope compositions, suggesting formation in isotopically distinct reservoirs. Refractory inclusions are ${ }^{16} \mathrm{O}$-enriched relative to chondrules but ${ }^{16} \mathrm{O}$-depleted relative to the Sun (McKeegan et al. 2011). The terrestrial fractionation line (TFL) and Carbonaceous Chondrite Anhydrous Mineral (CCAM) line are shown for reference. Data from Tenner et al. (2015), Schrader et al. (2014), Krot et al. (2006), and Makide et al. (2009) 
preservation of several nuclei in a chondrule melt. In contrast, non-porphyritic chondrules crystallised from melts formed by nearly complete melting of solid precursors and/or by gas-melt condensation.

Porphyritic chondrules commonly contain relict grains that did not crystallise from a host chondrule melt, and, therefore, provide constraints on the nature of chondrule precursor materials and chondrule-forming mechanism(s). Relict grains in chondrules can be distinguished based on their textures, mineralogy, chemical and oxygen isotopic compositions. Based on these characteristics, coarse-grained relict grains $(>10-100 \mu \mathrm{m})$ identified in porphyritic chondrules include CAIs, AOAs, fragments of chondrules of earlier generations (Fig. 6.2a, b), and, possibly fragments of thermally processed planetesimals (see Fig. 1 of Libourel and Krot 2007). Most relict ferromagnesian olivine and pyroxene grains have oxygenisotope compositions that differ from those of the host chondrule phenocrysts and mesostasis. However, the oxygen-isotope compositions of most relict olivine and pyroxene grains are generally similar to those of typical chondrules suggesting their close genetic relationship; these relict grains are most likely fragments of chondrules of earlier generations (Tenner et al. 2015; Schrader et al. 2014; Jones et al. 2004; Krot et al. 2005b; Russell et al. 2005; Kita et al. 2010; Rudraswami et al. 2011).

Because of high dissolutions rates of olivine in chondrule melts (Soulié et al. 2012), several micron-sized relict grains could have survived only in chondrules that experienced a very small degree of melting. Chondrules in CV, CR and ordinary chondrites are typically surrounded by finer-grained ferromagnesian silicate igneous rims (Krot and Wasson 1995; Krot et al. 2004). Oxidation states of igneous rims ( $\mathrm{Fa}$ or Fs contents in their olivines and pyroxenes) are generally similar to those of the host chondrules, suggesting formation under similar redox conditions. These rims appear to have formed by melting of relatively fine-grained solids $(<10 \mu \mathrm{m})$ that accreted on the surface of previously solidified chondrules (Krot and Wasson 1995), indicative or repeatable transient heating events experienced by chondrules. Like host chondrules, igneous chondrule rims typically contain relict grains (Nagashima et al. 2015).

\subsection{Chronology of Chondrule Formation}

\subsubsection{U-Corrected Pb-Pb Dating}

Of the various radiometric clocks, $\mathrm{U}$-corrected $\mathrm{Pb}-\mathrm{Pb}$ dating is the only method that provides a high-resolution assumption-free chronology of the first $10 \mathrm{Myr}$ of the Solar System. It is based on two isotopes of $\mathrm{U}$ that decay in a chain to stable $\mathrm{Pb}$ isotopes, namely ${ }^{235} \mathrm{U}$ to ${ }^{207} \mathrm{~Pb}$ with a half-life of $\sim 0.7 \mathrm{Gyr}$ and ${ }^{238} \mathrm{U}$ to ${ }^{206} \mathrm{~Pb}$ with a 
half-life of $\sim 4$ Gyr. This results in ${ }^{207} \mathrm{~Pb}_{\mathrm{R}} /{ }^{206} \mathrm{~Pb}_{\mathrm{R}}$ (where $\mathrm{R}=$ radiogenic) ratios that correspond to the amount of time passed since the system closed, by Eq. (6.1)

$$
\frac{{ }^{207} \mathrm{~Pb}_{\mathrm{R}}}{{ }^{206} \mathrm{~Pb}_{\mathrm{R}}}=\left(\frac{{ }^{235} \mathrm{U}}{{ }^{238} \mathrm{U}}\right)\left(\frac{e^{\lambda_{1} t}-1}{e^{\lambda_{2} t}-1}\right),
$$

where $\lambda_{1}$ and $\lambda_{2}$ are the decay constants for ${ }^{235} \mathrm{U}$ and ${ }^{238} \mathrm{U}$, respectively; and t represents time. The ${ }^{207} \mathrm{~Pb}_{\mathrm{R}} /{ }^{206} \mathrm{~Pb}_{\mathrm{R}}$ ratio of an inclusion is calculated by extrapolating from an array of measured $\mathrm{Pb}$ isotopic values in ${ }^{204} \mathrm{~Pb} /{ }^{206} \mathrm{~Pb}-{ }^{207} \mathrm{~Pb} /{ }^{206} \mathrm{~Pb}$ space that represent varying mixtures of radiogenic $\mathrm{Pb}$ and its initial $\mathrm{Pb}$ isotopic composition, which should approximate that of the Solar System's initial Pb isotope composition (Fig. 6.4). Thus, ages are defined through the internal isochron approach. It is apparent from Eq. (6.1) that knowledge of the ${ }^{238} \mathrm{U} /{ }^{235} \mathrm{U}$ of an object is required to accurately define its $\mathrm{Pb}-\mathrm{Pb}$ age. Although traditionally assumed to be 137.88 in all Solar System materials, the ${ }^{238} \mathrm{U} /{ }^{235} \mathrm{U}$ values of CAIs have been demonstrated to vary by approximately $0.35 \%$, which corresponds to offsets in calculated $\mathrm{Pb}-\mathrm{Pb}$ ages of up to 5 Myr (Brennecka et al. 2010). The observation of $U$ isotope variability, attributed to the decay of the short-lived ${ }^{247} \mathrm{Cm}$ nuclide $\left({ }^{247} \mathrm{Cm}\right.$ decays to ${ }^{235} \mathrm{U}$

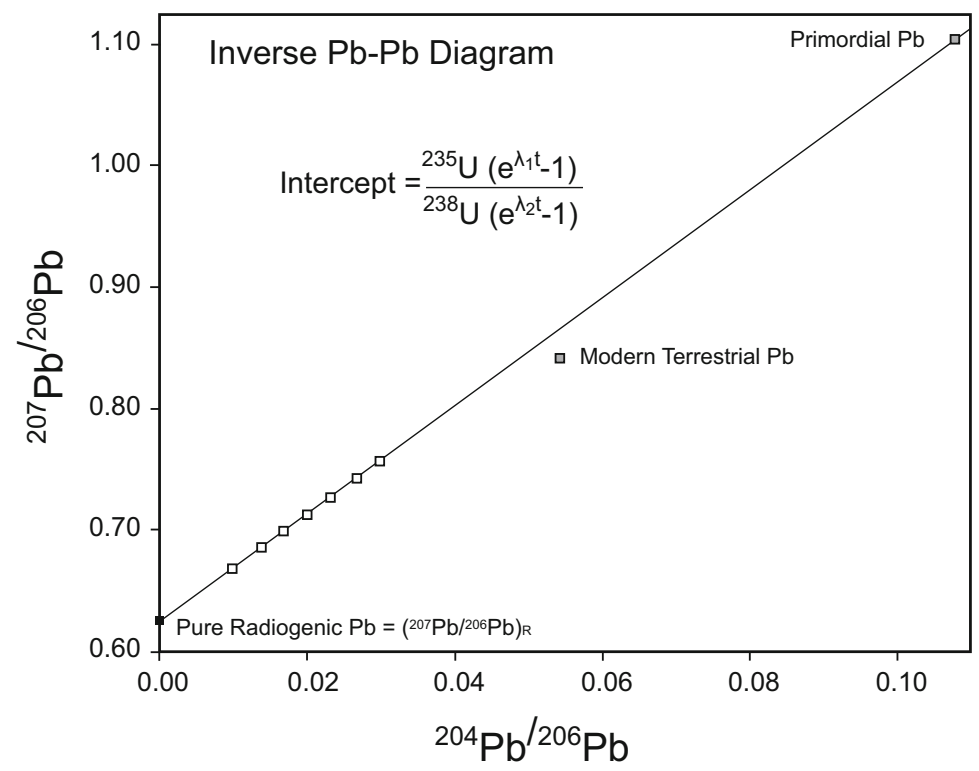

Fig. 6.4 Inverse $\mathrm{Pb}-\mathrm{Pb}$ diagram. The radiogenic ${ }^{207} \mathrm{~Pb} /{ }^{206} \mathrm{~Pb}$ ratio $\left[\left({ }^{207} \mathrm{~Pb} /{ }^{206} \mathrm{~Pb}\right)_{\mathrm{R}}\right]$ can be calculated by projecting a line through data points with variable mixtures of radiogenic $\mathrm{Pb}$ and initial $\mathrm{Pb}$ to the $\mathrm{y}$ intercept, where the initial $\mathrm{Pb}$ is theoretically zero.This variability is created by strategically analysing related fragments, minerals, or acid leachates with variable $\mathrm{U} / \mathrm{Pb}$ ratios. (Subscript $\mathrm{R}$ refers to radiogenic; $\lambda_{1}$ and $\lambda_{2}$ represent the decay constants of ${ }^{235} \mathrm{U}$ and ${ }^{238} \mathrm{U}$, respectively; and $t$ represents time) 
with a half-life of $\sim 15.6 \mathrm{Myr}$ ), voided all published $\mathrm{Pb}-\mathrm{Pb}$ ages for Solar System materials that were based on an assumed ${ }^{238} \mathrm{U} /{ }^{235} \mathrm{U}$ ratio and made clear the need to have measurements of the $\mathrm{U}$ isotopic compositions for all materials dated by the $\mathrm{Pb}-\mathrm{Pb}$ method. However, more recent work suggests that the ${ }^{238} \mathrm{U} /{ }^{235} \mathrm{U}$ variability may be limited to the CAI reservoir, where $\mathrm{Cm} / \mathrm{U}$ fractionation or, alternatively, mass-dependent ${ }^{238} \mathrm{U} /{ }^{235} \mathrm{U}$ variations can occur via evaporation and/or condensation processes (Connelly et al. 2012; Tissot et al. 2016). This view is supported by the homogeneous ${ }^{238} \mathrm{U} / 235 \mathrm{U}$ of chondrules and bulk planetary materials, which is used to define the solar ${ }^{238} \mathrm{U} /{ }^{235} \mathrm{U}$ value of 137.786 (Connelly et al. 2012; Brennecka et al. 2015).

The majority of chondrules are believed to have formed by melting of disk dust and rapidly (10-1000 $\mathrm{K} \mathrm{h}^{-1}$ ) cooled (Scott 2007). The so-called nebular chondrules are taken here as representing chondrules formed within the protoplanetary disk before dust and gas dissipation. A second group of chondrules apparently formed later by planetary collisions, namely chondrules from the metal-rich $\mathrm{CB}$ chondrites. Initial attempts to date nebular chondrules pooled a number of objects that resulted in ages that were $4563.66 \pm 0.63$ (Amelin et al. 2002) and $4564.32 \pm 0.81 \mathrm{Myr}$ (Connelly and Bizzarro 2009) (both ages adjusted for a U isotopic composition of 137.786). These ages can only reflect the average age of the chondrules pooled for these studies. Large chondrules from the Gujba CB chondrite, believed to have formed by a collision between two planetesimals, have been dated as individual inclusions with an average age of 4562.49 $\pm 0.21 \mathrm{Myr}$ (Bollard et al. 2015).

The only published report on the $\mathrm{U}$-corrected $\mathrm{Pb}-\mathrm{Pb}$ ages of individual nebular chondrules indicates a protracted formation history, with an age range for five chondrules from $4567.32 \pm 0.42 \mathrm{Myr}$ to $4564.71 \pm 0.30 \mathrm{Myr}$ (Connelly et al. 2012) (Fig. 6.5). The isochrons for all five chondrules project back to an initial $\mathrm{Pb}$ isotopic composition that is close to the presumed primordial isotopic composition of the Solar System, but with some variability interpreted to reflect a complex formation history involving more than one melting event for some chondrule precursors. In all cases, the isochrons projected well above the field for modern terrestrial $\mathrm{Pb}$ indicating that this was not the source of non-radiogenic $\mathrm{Pb}$ in the fractions used to define the isochrons. This study concluded that the ages derived for the five welldefined isochrons with sensible extrapolations to initial $\mathrm{Pb}$ compositions represent the age of the last crystallisation of these chondrules. They ranged in ages from the oldest chondrule that overlaps the brief formation age of CAIs to $\sim 2.6 \mathrm{Myr}$ after. Thus, the first chondrules formed contemporaneously with CAIs and the energy source responsible for the formation of chondrules lasted for at least $\sim 2.6 \mathrm{Myr}$ following condensation of CAIs. A subsequent study of 17 individual chondrules from CR and ordinary chondrites has confirmed the oldest ages of chondrules and extends the age range of nebular chondrules formation to $\sim 3.6 \mathrm{Myr}$ after CAIs (Bollard et al. 2017). The timescales for the melting of disk solids inferred from the $\mathrm{Pb}-\mathrm{Pb}$ dates is comparable to the $\sim 3 \mathrm{Myr}$ median lifetime of disks around low-mass stars indicated by astronomical observations of young stellar objects within starforming regions (Evans et al. 2009). Thus, the formation of chondrules may reflect a generic process intrinsically linked to the secular evolution of accretionary disks 


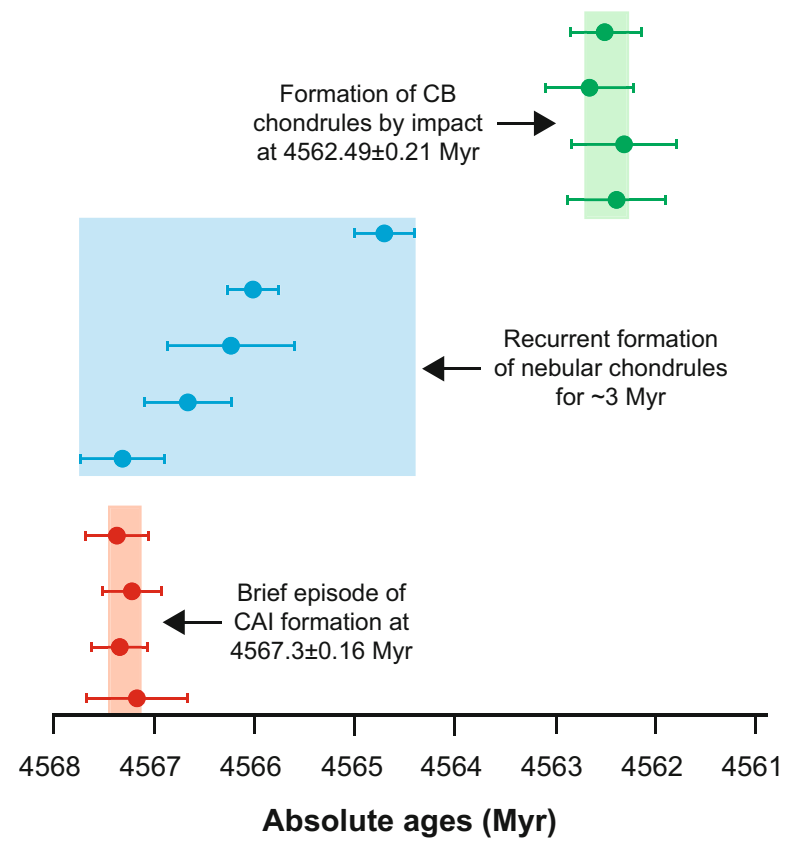

Fig. 6.5 U-corrected $\mathrm{Pb}-\mathrm{Pb}$ ages of individual chondrules and CAIs from various carbonaceous and ordinary chondrites. Figure modified from Connelly et al. (2012)

not unique to our Solar System. If chondrules are indeed an important ingredient promoting the growth of asteroids and planetary embryos, the U-corrected $\mathrm{Pb}-\mathrm{Pb}$ dates indicate that they existed in the disk over the timescale required to assemble Mars-sized objects.

\subsubsection{The ${ }^{26} \mathrm{Al}-{ }^{26} \mathrm{Mg}$ Decay System}

With a half-life of $\sim 705,000$ years (Norris et al. 1983 ), the ${ }^{26} \mathrm{Al}-\mathrm{to}-{ }^{26} \mathrm{Mg}$ decay system is one of the most widely used relative chronometers to understand solid formation in the early Solar System. Indeed, the short-half life of ${ }^{26} \mathrm{Al}$ coupled to significant $\mathrm{Al} / \mathrm{Mg}$ fractionation by condensation and evaporation processes as well as melting and solidification allows for the determination of highly precise relative ages. For example, it has been proposed that the epoch of primary condensation of CAIs from CV chondrites may have been as short as $~ 4500$ years based on the ${ }^{26} \mathrm{Al}-{ }^{26} \mathrm{Mg}$ system (Larsen et al. 2011; Jacobsen et al. 2008). These objects define the canonical ${ }^{26} \mathrm{Al} /{ }^{27} \mathrm{Al}$ ratio of $\sim 5 \times 10^{-5}$, which is widely thought to reflect the initial ${ }^{26} \mathrm{Al}$ abundance of the Solar System. Some CAIs define lower initial ${ }^{26} \mathrm{Al} /{ }^{27} \mathrm{Al}$ ratios (Fig. 6.6), which may reflect secondary disturbance of the ${ }^{26} \mathrm{Al}-{ }^{26} \mathrm{Mg}$ system, 


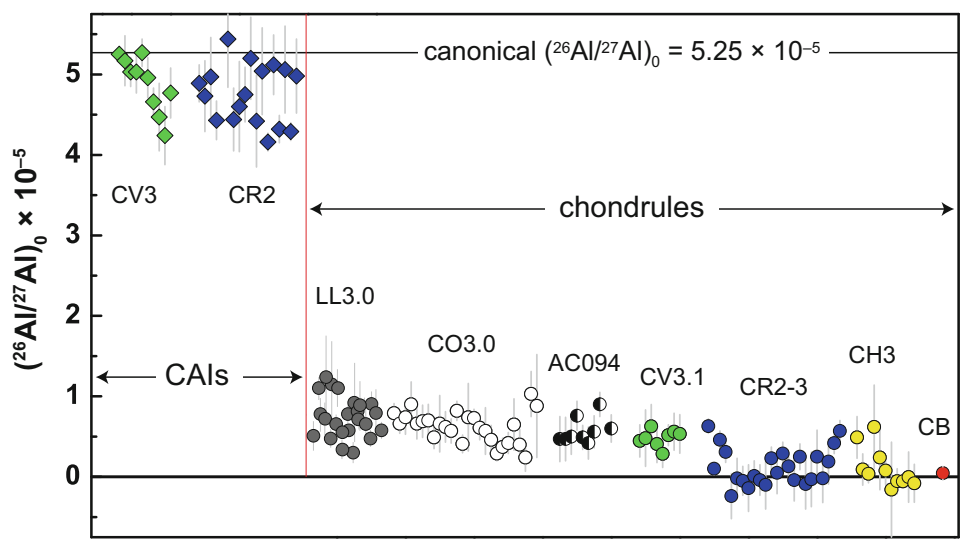

Fig. 6.6 The inferred initial ${ }^{26} \mathrm{Al} /{ }^{27} \mathrm{Al}$ ratios $\left[\left({ }^{26} \mathrm{Al} /{ }^{27} \mathrm{Al}\right)_{0}\right]$ in igneous CAIs and chondrules in the least metamorphosed ordinary and carbonaceous chondrites. CAIs record $\left({ }^{26} \mathrm{Al} /{ }^{27} \mathrm{Al}\right)_{0}$ of $4-5 \times 10^{-5}$. In contrast, the $\left({ }^{26} \mathrm{Al} /{ }^{27} \mathrm{Al}\right)_{0}$ of chondrules are systematically lower than in CAIs, $<1.5 \times 10^{-5}$. The lower initial is interpreted as reflecting a reduced initial abundance of ${ }^{26} \mathrm{Al}$ in the precursor material of chondrules relative to the CAI-forming region. AC094, Acfer 094. Data from Makide et al. (2009), Villeneuve et al. (2009), Larsen et al. (2011), Hutcheon and Hutchison (1989), Kita et al. (2000, 2013), Kita and Ushikubo (2012), Kunihiro et al. (2004), Kurahashi et al. (2008), MacPherson et al. (2012), Olsen et al. (2013), Ushikubo et al. (2013), and Krot and Nagashima (2017)

or alternatively, remelting events within $\sim 300,000$ years of CAI condensation (MacPherson et al. 2012). In contrast, chondrules recorded systematically lower initial ${ }^{26} \mathrm{Al} /{ }^{27} \mathrm{Al}$ ratios. Assuming that the canonical ${ }^{26} \mathrm{Al} /{ }^{27} \mathrm{Al}$ ratio represents initial abundance of ${ }^{26} \mathrm{Al}$ for the Solar System as a whole, it is possible to derive a relative chronology of chondrule formation with respect to the time of condensation of $\mathrm{CV}$ CAIs. Using this approach, a number of studies have suggested a time delay of $\sim 1-$ $2 \mathrm{Myr}$ between the formation of CAIs and chondrules, a period commonly referred to as the CAI-chondrule age gap (Krot et al. 2009). Such an age gap, however, is not apparent in the $\mathrm{U}$-corrected $\mathrm{Pb}-\mathrm{Pb}$ ages of individual chondrules.

Luu et al. (2015) recently suggested that the bulk Al-Mg systematics of a number of chondrules from the non-pristine and aqueously altered Allende carbonaceous chondrites can be used to date the timing of condensation of chondrule precursors. This is based on the observations that a number of chondrules form an array in the Al-Mg diagram that corresponds to an initial ${ }^{26} \mathrm{Al} /{ }^{27} \mathrm{Al}$ value of $1.2 \times 10^{-5}$, which the authors interpret as reflecting the timing of cessation of condensation of chondrule precursors $\sim 1.5 \mathrm{Myr}$ after formation of the Solar System first solids. This conclusion, however, is based on the assumption of ${ }^{26} \mathrm{Al}$ homogeneity, which has not been demonstrated by any study and is not constrained by their data. Moreover, the linear relationship is essentially defined by Al-rich chondrules, which are known to contain a recycled CAI component in the Allende meteorite (Krot et al. 2009). Thus, it is unlikely that the array defines a meaningful isochron. We suggest that the acceptable MSWD (mean square weighted deviations) defined by the linear 
array is a results of the low precision of the $\mathrm{Mg}$-isotope data relative to the stateof-the-art (Bizzarro et al. 2011), which is a factor of 10 better. As such, higher precision data is required to better understand the significance of this array. Finally, we note that the initial ${ }^{26} \mathrm{Al} /{ }^{27} \mathrm{Al}$ value of $1.2 \times 10^{-5}$ defined by the bulk Allende chondrules is comparable to the initial ${ }^{26} \mathrm{Al} /{ }^{27} \mathrm{Al}$ value of $1.33 \times 10^{-5}$ proposed by Schiller et al. (2015a) as the initial ${ }^{26} \mathrm{Al}$ abundance for the inner protoplanetary disk. In this interpretation, the bulk Allende chondrule isochron may represent the timing of formation of chondrule precursors contemporaneously with CAI formation.

A number of recent studies have cast serious doubts on the assumption of ${ }^{26} \mathrm{Al}$ homogeneity, suggesting instead that initial heterogeneity in the ${ }^{26} \mathrm{Al} /{ }^{27} \mathrm{Al}$ ratioperhaps up to $80 \%$ of the canonical value-may have existed throughout the inner Solar System (Larsen et al. 2011; Schiller et al. 2015a). In particular, Schiller et al. (2015a) recently provided a detailed comparison of the U-corrected $\mathrm{Pb}-\mathrm{Pb}$ and internal ${ }^{26} \mathrm{Al}-{ }^{26} \mathrm{Mg}$ isochron ages for three rapidly cooled angrite meteorites. Their results demonstrate that the ${ }^{26} \mathrm{Al}-{ }^{26} \mathrm{Mg}$ ages obtained for angrites are systematically younger by $\sim 1.5 \mathrm{Myr}$ relative to their absolute ages, establishing that the angrite parent body accreted from precursor material typified by a reduced initial abundance of ${ }^{26} \mathrm{Al}$ relative to the canonical ratio. The three angrites concordantly define an initial ${ }^{26} \mathrm{Al} /{ }^{27} \mathrm{Al}$ of $1.33_{-0.18}^{+0.21} \times 10^{-5}$ for the precursor of their parent body, which is identical to the estimate of $1.61 \pm 0.32 \times 10^{-5}$ inferred from the $\mu^{26} \mathrm{Mg}^{*}$ compositions of young angrites (Larsen et al. 2011). Preliminary reports on the comparison of the U-corrected $\mathrm{Pb}-\mathrm{Pb}$ and internal ${ }^{26} \mathrm{Al}-{ }^{26} \mathrm{Mg}$ isochron ages of individual chondrules from various chondrites also yield systematically younger ${ }^{26} \mathrm{Al}-{ }^{26} \mathrm{Mg}$ ages relative to the $\mathrm{Pb}-\mathrm{Pb}$ dates (Bizzarro et al. 2014). Collectively, these data support the view that the bulk of the material that accreted to form asteroidal bodies and planetary embryos was characterised by a reduced initial ${ }^{26} \mathrm{Al} /{ }^{27} \mathrm{Al}$ ratios relative to the canonical value. The reduced inner Solar System initial abundance of ${ }^{26} \mathrm{Al}$ relative to that of CAIs has been interpreted as reflecting the thermal processing and unmixing of presolar components with contrasting thermal properties (Trinquier et al. 2009; Paton et al. 2013; Schiller et al. 2015b). In addition, it has been proposed that bodies accreted beyond the orbits of the gas giants contain significant amount of ${ }^{26} \mathrm{Al}$-free, thermally unprocessed molecular cloud matter (Van Kooten et al. 2016). Given the mounting evidence for initial ${ }^{26} \mathrm{Al}$ heterogeneity, it appears unlikely that the ${ }^{26} \mathrm{Al}-{ }^{26} \mathrm{Mg}$ system can provide an accurate chronology of the early Solar System. In this respect, the apparent $\sim 1-2 \mathrm{Myr}$ age gap inferred from the ${ }^{26} \mathrm{Al}-{ }^{26} \mathrm{Mg}$ system may simply reflect a reduced initial ${ }^{26} \mathrm{Al}$ abundance in chondrule precursors, which would bring the chondrule ${ }^{26} \mathrm{Al}-{ }^{26} \mathrm{Mg}$ age distribution in line with the U-corrected $\mathrm{Pb}-\mathrm{Pb}$ dates (Bollard et al. 2017).

\subsubsection{The ${ }^{182} \mathrm{Hf}_{-}{ }^{182}$ W Decay System}

The ${ }^{182} \mathrm{Hf}$ short-lived radionuclide decays to ${ }^{182} \mathrm{~W}$ with a half-life of $\sim 9 \mathrm{Myr}$. Due to the contrasting geochemical behaviour of Hf (lithophile) and W (siderophile), 
these two elements are fractionated by silicate-metal segregation processes that occurred during the accretion, differentiation and early evolution of asteroidal bodies, planetary embryos and planets (Kleine et al. 2009). In contrast to ${ }^{26} \mathrm{Al}$, which requires late-stage addition of stellar debris to the Sun's parental molecular cloud, the initial Solar System inventory of ${ }^{182} \mathrm{Hf}$ is believed to reflect long-term, steady-state galactic stellar nucleosynthesis before the formation of the protosolar molecular cloud (Wasserburg et al. 2006; Holst et al. 2013). If correct, this supports the view that the ${ }^{182} \mathrm{Hf}$ nuclide was homogeneously distributed in the protoplanetary disk at the time of formation of canonical CAIs. However, given the low abundance of $\mathrm{W}$ in chondritic components such as chondrules, it is not possible to date individual objects thereby necessitating the pooling of a significant amount of chondrules to obtain sufficient mounts of W. Using this approach, Budde et al. (2016) recently attempted to provide a chronology of the Allende CV chondrite chondrule formation based on the ${ }^{182} \mathrm{Hf}-{ }^{182} \mathrm{~W}$ system. In particular, these authors inferred that the $\sim 3 \mathrm{Myr}$ duration of chondrule formation documented by Connelly et al. (2012) is inconsistent with presumed chemical and isotopic complementary between chondrules and matrix that supports models of local and brief chondrule formation (Hezel and Palme 2010; Palme et al. 2015; Ebel et al. 2016). They hypothesised that the $\mathrm{Pb}-\mathrm{Pb}$ ages reflect late stage parent body alteration without providing any specific mechanism to explain isochrons as old as CAIs. Instead, they used the short-lived ${ }^{182} \mathrm{Hf}^{182} \mathrm{~W}$ decay system applied to $100 \mathrm{~s}$ or $1000 \mathrm{~s}$ of chondrules as well as matrix and bulk samples in an attempt to obtain the true age range of chondrules. A similar approach was used by Becker et al. (2015). However, using matrix, bulk samples and bulk chondrules to define ${ }^{182} \mathrm{Hf}-{ }^{182} \mathrm{~W}$ isochrons requires independent evidence for a single, closed-system Hf-W fractionation event affecting the matrix and chondrules to fulfil the basic requirement of a meaningful isochron.

Budde et al. (2016) used arguments for complementarity between chondrules and matrix, including their own ${ }^{183} \mathrm{~W}$ data, to infer a brief formation interval for chondrules and their immediate accretion to form chondrites with cogenetic matrix. Invoking this model to infer coeval fractionation of $\mathrm{Hf}$ and $\mathrm{W}$ between chondrules and matrix, these authors used these entities and mixtures of them to define a Hf-W array that corresponds to an age of $2.2 \pm 0.8 \mathrm{Myr}$ after the formation of CAIs that they accept as the time window of chondrule formation. This overlaps the result of Becker et al. (2015) who defined a chrondrule formation window of $-0.9 \pm 2.8 \mathrm{Myr}$ relative to the formation age of CAIs. However, a recent $\mathrm{Mg}$ and $\mathrm{Cr}$ isotope study of individual $\mathrm{CV}$ chondrules does not support the required complementarity between matrix and chondrules (Olsen et al. 2016). Furthermore, linearity of large multichondrule fractions in Hf-W space is predicted if the age distributions of the various populations are similar, even if they are not coeval. In this case, their age would approximate the average age of chondrules if the $\mathrm{Hf} / \mathrm{W}$ ratio of the matrix is close to the composition of the bulk Solar System. Finally, we note that two earlier studies (Amelin and Krot 2007; Connelly et al. 2008) have investigated the $\mathrm{Pb}-\mathrm{Pb}$ isotopic dates of Allende chondrule populations to derive average ages for chondrule formation of $1.67 \pm 0.9 \mathrm{Myr}$ and $2.85 \pm 0.45 \mathrm{Myr}$ after CAI formation [calculated 
using ${ }^{238} \mathrm{U} /{ }^{235} \mathrm{U}$ of 137.786 (Connelly et al. 2012)]. These ages are consistent with that reported by Budde et al. (2016). This may indicate that both systems remained closed in each chondrule after their respective final nebular heating event and, therefore, both are capable of returning primary age information about chondrules formation, even in an aqueously altered and metamorphosed meteorite like Allende. However, only the $\mathrm{Pb}-\mathrm{Pb}$ system is capable of dating individual chondrules to determine the true age range of nebular chondrule formation.

\subsection{Mechanism and Style of Asteroidal Accretion}

The short time interval inferred for the formation of chondrules within individual chondrite groups based on the ${ }^{26} \mathrm{Al}-{ }^{26} \mathrm{Mg}$ system can be used to argue for a rapid accretion of chondrite parent bodies. In this model, chondrules formed in highdensity regions that were possibly self-gravitating, which resulted in the rapid collapse and accretion of these objects into chondritic parent bodies (Alexander et al. 2008). This implies that chondrule formation and asteroidal accretion are intrinsically linked processes. However, the protracted timescale for the formation of chondrules inferred from the assumption-free $\mathrm{U}$-corrected $\mathrm{Pb}-\mathrm{Pb}$ dating method (Connelly et al. 2012; Bollard et al. 2017) is inconsistent with this model. Indeed, the $\sim 3$ Myr formation interval for chondrules from various chondrite groups indicates prolonged accretion timescales for chondritic parent bodies.

Recent numerical simulations suggest that the formation of asteroidal bodies and planetary embryos may be a two-step process, where first generation planetesimal seeds of $\sim 50 \mathrm{~km}$ diameter form rapidly via streaming instabilities (Johansen et al. 2007) followed by the protracted gas-drag-assisted accretion of chondrules during the lifetime of the protoplanetary disk (Johansen et al. 2015). The gas-drag assisted accretion of chondrules onto planetesimals is a process analogous to pebble accretion, which is the accretion of centimetre- to metre-sized particles loosely bound to the gas onto planetesimals seeds (Bitsch et al. 2015). In these simulations, the largest planetesimals of a population with a characteristic radius of $\sim 100 \mathrm{~km}$ undergo run-away accretion of chondrules forming Mars-sized planetary embryos within a timescale of $\sim 3-5 \mathrm{Myr}$. This timescale is in agreement with the timing of formation and differentiation of Mars inferred from ${ }^{182} \mathrm{Hf}^{-182} \mathrm{~W}$ chronology (Dauphas and Pourmand 2011).

A model of continuous asteroidal accretion during the lifetime of the protoplanetary disk has important implications for the thermal evolution of asteroidal bodies given that the accretion process is completed beyond the time when ${ }^{26} \mathrm{Al}$ can provide enough energy to induce heating and differentiation (Larsen et al. 2016). Thus, protracted asteroidal accretion predicts the existence of partially differentiated asteroidal bodies, namely onion-shell structured bodies with differentiated interiors consisting of silicate mantles and metallic cores surrounded by unmelted chondritic crusts (Weiss and Elkins-Tanton 2013). Although controversial, this proposal is 
apparently supported by the discovery of remnant magnetism in chondritic meteorites suggesting the existence of dynamo field (Carporzen et al. 2011), which can only occur through the establishment of a convecting metallic core.

\subsection{Accretion Regions of Chondrite Parent Bodies}

Large-scale nucleosynthetic isotopic heterogeneity exists among inner Solar System solids, planets, and asteroids, most noticeably for neutron-rich isotopes of the iron-group elements such as ${ }^{48} \mathrm{Ca},{ }^{50} \mathrm{Ti},{ }^{54} \mathrm{Cr}$ and ${ }^{62} \mathrm{Ni}$ (Birck 2004). In particular, significant ${ }^{54} \mathrm{Cr}$ variability has been documented between carbonaceous chondrite groups and meteorites originating from asteroidal bodies believed to have formed in the accretion regions of terrestrial planets such as ordinary and enstatite chondrites and the majority of differentiated meteorites (Fig. 6.7). Bulk carbonaceous chondrites are characterised by excesses in $\mu^{54} \mathrm{Cr}$ ranging from $+57 \pm 11$ to $+156 \pm 6 \mathrm{ppm}$ relative to the terrestrial composition (Trinquier et al. 2007). In contrast, enstatite and ordinary chondrites as well as Mars, the Moon and most differentiated

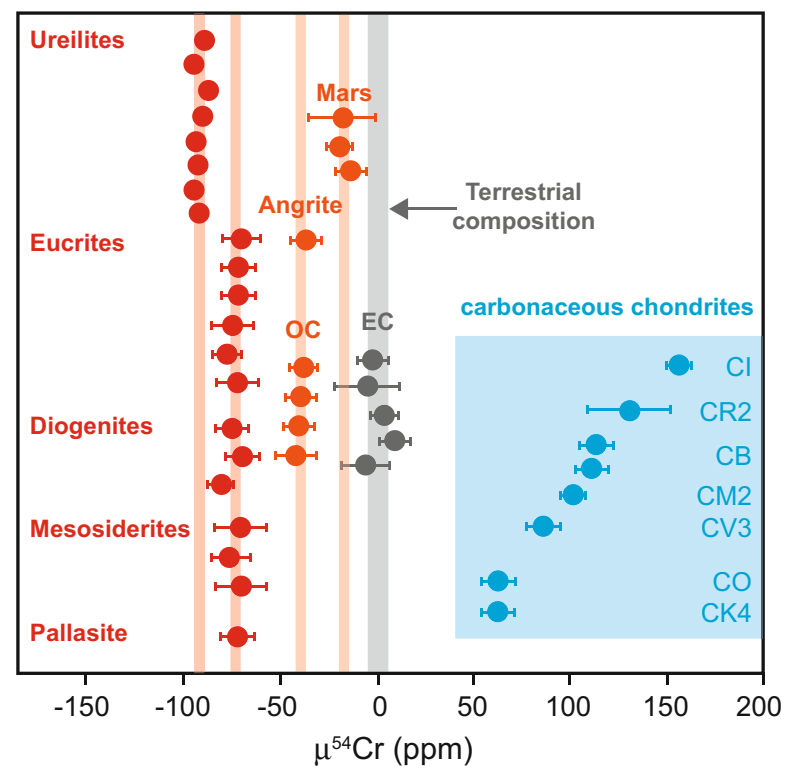

Fig. 6.7 $\mu^{54} \mathrm{Cr}$ composition of bulk planetary reservoirs. The $\mu^{54} \mathrm{Cr}$ notation reflects per $10^{6}$ deviations of the ${ }^{54} \mathrm{Cr} /{ }^{52} \mathrm{Cr}$ ratio of a sample relative to the terrestrial composition. Carbonaceous chondrites formed from material that is distinct from the bulk of the material thought to have accreted in the terrestrial planet region. Thus, the $\mu^{54} \mathrm{C}$ values can be used to track genetic relationships between early solids and bulk Solar System reservoirs. $E C$, enstatite chondrites. $O C$, ordinary chondrites. Uncertainties for ureilite data are smaller than symbols. Data from Trinquier et al. (2007) and Yamakawa et al. (2010) 
meteorites record $\mu^{54} \mathrm{Cr}$ values that span from the terrestrial composition to deficits of $\sim 100 \mathrm{ppm}$. Thus, carbonaceous chondrites have apparently sampled, on average, material formed in a distinct reservoir in the terms of their $\mu^{54} \mathrm{Cr}$ composition compared to the enstatite and ordinary chondrite population. The dichotomy in ${ }^{54} \mathrm{Cr}$ composition observed between carbonaceous and non-carbonaceous material is also mirrored by a number of other nuclides, including ${ }^{43} \mathrm{Ca},{ }^{48} \mathrm{Ca},{ }^{46} \mathrm{Ti},{ }^{50} \mathrm{Ti},{ }^{62} \mathrm{Ni}$ and ${ }^{88} \mathrm{Sr}$ (Paton et al. 2013; Schiller et al. 2015b; Regelous et al. 2008; Trinquier et al. 2009). This variability, which is interpreted as reflecting the selective unmixing of nucleosynthetic components during the earliest stages of Solar System formation (Trinquier et al. 2009; Paton et al. 2013; Schiller et al. 2015b; Van Kooten et al. 2016), provides a means of probing genetic relationships between early formed solids, asteroids and planetary bodies.

It has been suggested that the observed dichotomy in the abundance of the ${ }^{54} \mathrm{Cr}$ tracer between carbonaceous and non-carbonaceous chondrites essentially reflects distinct accretion regions of their parent bodies, namely that the carbonaceous material formed beyond the snow line whereas the non-carbonaceous material originated Sunward of the snow line (Larsen et al. 2016; Warren 2011). Accretion of the carbonaceous chondrites beyond the snow line is in accord with the much higher water content of these meteorites (Robert and Epstein 1982; Kerridge 1985) compared to that of enstatite and ordinary chondrites (McNaughton et al. 1981; Robert et al. 1987; Hutson and Ruzicka 2000). Moreover, recent dynamical models for the early evolution of the Solar System suggest that the parent asteroids of carbonaceous chondrites formed between and beyond the accretion regions of the giant planets and were implanted in the asteroid belt following the final outward migration of Jupiter (Walsh et al. 2011).

In the inner Solar System, bulk planetary materials with solar or near-solar ${ }^{27} \mathrm{Al} /{ }^{24} \mathrm{Mg}$ ratios record positively correlated variability in $\mu^{26} \mathrm{Mg} *$ and $\mu{ }^{54} \mathrm{Cr}$. This correlation is interpreted as reflecting progressive thermal processing of in-falling ${ }^{26} \mathrm{Al}$-rich molecular cloud material, which resulted in preferential loss by sublimation of thermally unstable and isotopically anomalous presolar carriers, producing residual isotopic heterogeneity (Trinquier et al. 2009; Paton et al. 2013; Schiller et al. 2015b; Van Kooten et al. 2016). In this model, the correlated $\mu^{26} \mathrm{Mg}^{*}-\mu^{54} \mathrm{Cr}$ array represents the unmixing of distinct dust populations with contrasting thermal properties, namely unmixing of old, galactically inherited homogeneous dust from a young supernovae-derived dust component formed shortly prior to or during the evolution of the giant molecular cloud parental to the protosolar molecular cloud core. We illustrate this concept in Fig. 6.8, which depicts the $\mu^{26} \mathrm{Mg}^{*}-\mu^{54} \mathrm{Cr}$ systematics of three reservoirs, namely a bulk Solar System reservoir, the CAIforming gas and a residual disk solid reservoir. Thermal processing of dust of solar composition occurs at $\mathrm{T}_{1}$, which results in the establishment of two complementary reservoirs, namely the $\mathrm{CAI}$ forming gas enriched in ${ }^{26} \mathrm{Al}$ and ${ }^{54} \mathrm{Cr}$ and a residual disk solid reservoir depleted in ${ }^{26} \mathrm{Al}$ and ${ }^{54} \mathrm{Cr}$ relative to the bulk solar composition. The timing of the main thermal processing event $\left(T_{1}\right)$ is thought to have occurred shortly prior to CAI condensation. Following their establishment, the $\mu^{26} \mathrm{Mg}^{*}$ isotopic 


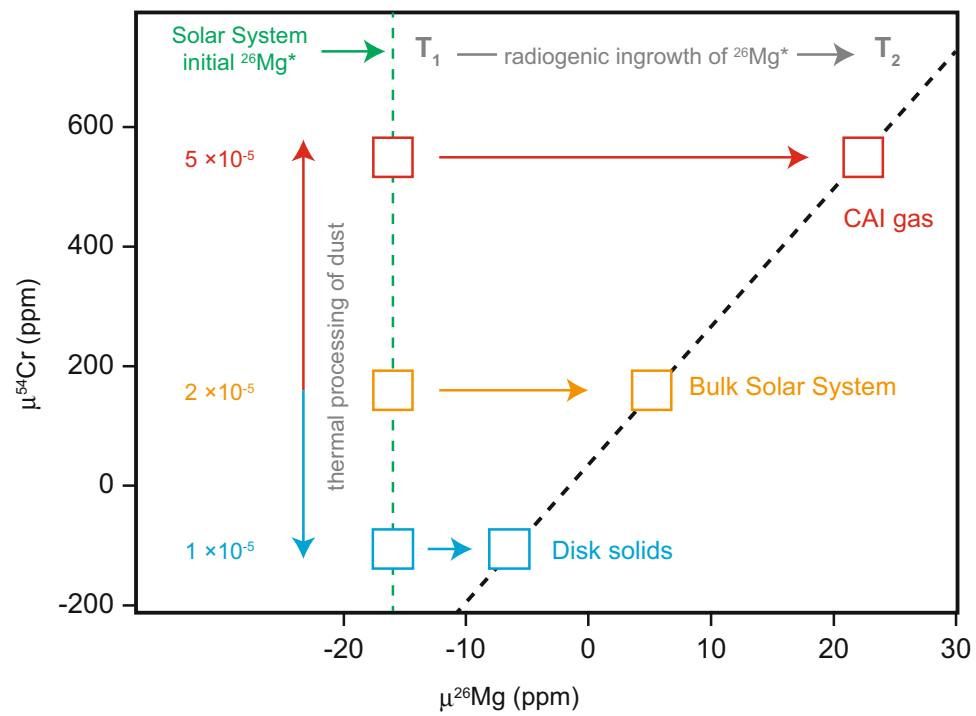

Fig. 6.8 $\mu^{26} \mathrm{Mg}^{*}-\mu^{54} \mathrm{Cr}$ variation diagram. Diagram depicting the effect of early thermal processing of presolar components on the $\mu^{26} \mathrm{Mg}^{*}$ and $\mu^{54} \mathrm{Cr}$ isotope composition of early Solar System reservoirs with solar ${ }^{27} \mathrm{Al} /{ }^{24} \mathrm{Mg}$ ratios. A thermal processing event occurs at $\mathrm{T}_{1}$, which leads to enrichments in ${ }^{26} \mathrm{Al}$ and ${ }^{54} \mathrm{Cr}$ in a hypothetical gas and depletion in ${ }^{26} \mathrm{Al}$ and ${ }^{54} \mathrm{Cr}$ in the residual disk solids. The $\mu^{26} \mathrm{Mg}$ * compositions of the reservoirs then evolve according to their initial ${ }^{26} \mathrm{Al}$ inventory to their present-day compositions at time $\mathrm{T}_{2}$, generating a positively correlated array

composition of the respective reservoirs evolve according to their initial ${ }^{26} \mathrm{Al} /{ }^{27} \mathrm{Al}$ to their present-day, measured compositions $\left(\mathrm{T}_{2}\right)$. Thermal processing at early times thus results in a positively correlated array in the measured $\mu^{26} \mathrm{Mg} *$ and $\mu^{54} \mathrm{Cr}$ compositions of bulk planetary materials with solar or near-solar ${ }^{27} \mathrm{Al} /{ }^{24} \mathrm{Mg}$ ratios.

Van Kooten et al. (2016) recently proposed that the metal-rich carbonaceous chondrites ( $\mathrm{CB}, \mathrm{CH}$ and $\mathrm{CR})$ accreted from material predominately located beyond the orbits of the gas giant planets. This proposal is based on the coupled $\mu{ }^{54} \mathrm{Cr}$ and $\mu^{26} \mathrm{Mg} *$ compositions of metal-rich carbonaceous chondrites and their components, which is distinct from bulk inner Solar System objects. In detail, metal-rich carbonaceous chondrites and their components do not plot on the Solar System's $\mu^{54} \mathrm{Cr}-\mu{ }^{26} \mathrm{Mg}$ * correlation line but instead have a unique isotopic signature extending from an inner Solar System composition toward a ${ }^{26} \mathrm{Mg}^{*}$-depleted and ${ }^{54} \mathrm{Cr}$-enriched component (Fig. 6.9). This composition is consistent with that expected for thermally unprocessed primordial molecular cloud material before its pollution by stellar-derived ${ }^{26} \mathrm{Al}$. Moreover, Van Kooten et al. (2016) suggest that the $\mu^{54} \mathrm{Cr}$ and $\mu^{26} \mathrm{Mg} *$ compositions of these objects require significant amounts (25$50 \%$ ) of primordial molecular cloud matter in their precursor material. Given that such high fractions of primordial molecular cloud material are expected to survive only in the outer Solar System, the authors infer that, similarly to cometary bodies, metal-rich carbonaceous chondrites are samples of planetesimals that accreted 

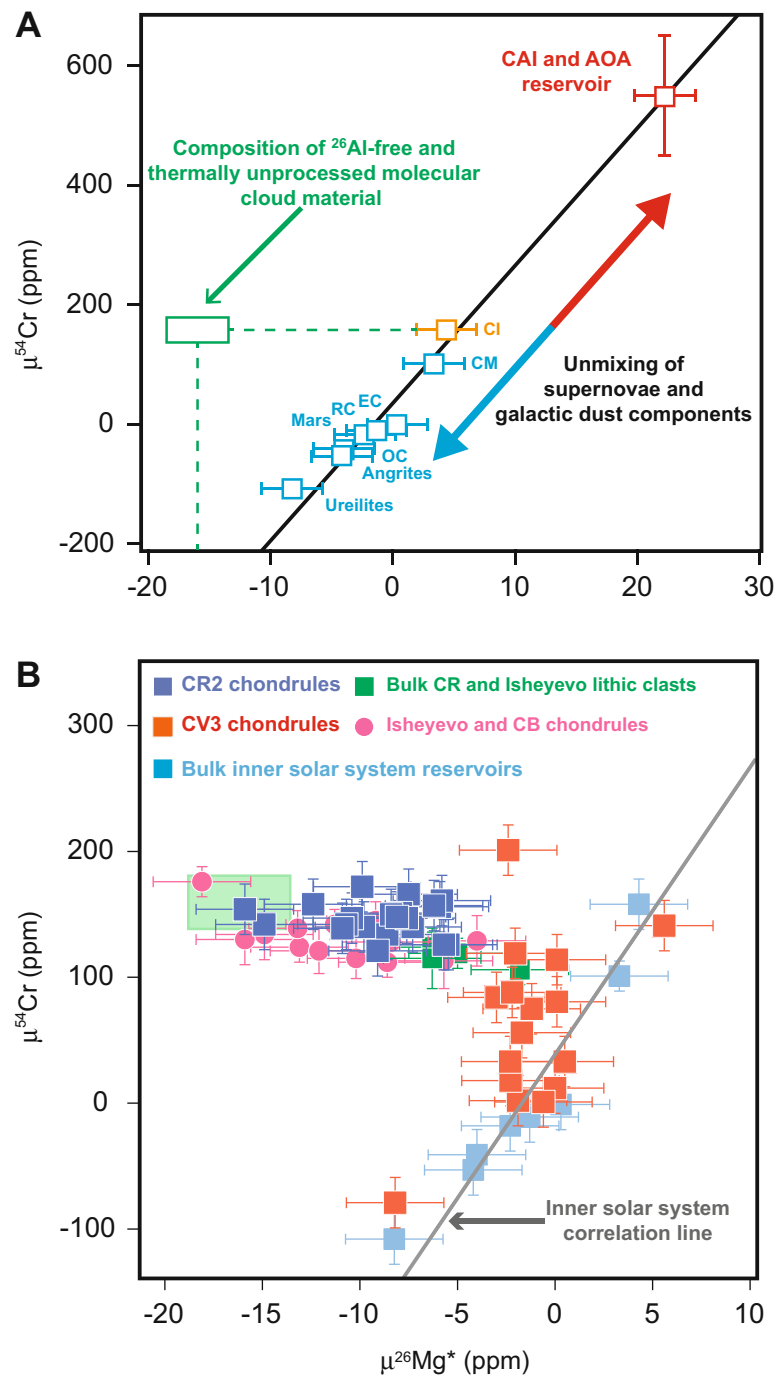

Fig. 6.9 $\mu^{26} \mathrm{Mg}^{*}-\mu^{54} \mathrm{Cr}$ variation diagrams. (a) Inner Solar System $\mu^{26} \mathrm{Mg} *-\mu^{54} \mathrm{Cr}$ correlation. $A O A$, amoeboid olivine aggregates; $E C$, enstatite chondrites; $O C$, ordinary chondrites; $R C$, Rumuruti chondrites. The $\mu^{26} \mathrm{Mg}^{*}$ and $\mu^{54} \mathrm{Cr}$ compositions of the ${ }^{26} \mathrm{Al}$-free and thermally unprocessed molecular cloud material are defined by CI chondrites and the initial $\mu^{26} \mathrm{Mg}^{*}$ value of the Solar System. (b) The $\mu^{26} \mathrm{Mg}^{*}$ and $\mu^{54} \mathrm{Cr}$ compositions of metal-rich chondrites and their components. The green box represents the predicted composition of the ${ }^{26} \mathrm{Al}$-free and thermally unprocessed molecular cloud material. Modified from Van Kooten et al. (2016) and Olsen et al. (2016)

beyond the orbits of the gas giants. Collectively, the coupled $\mu^{54} \mathrm{Cr}-\mu^{26} \mathrm{Mg}$ * systematics of various chondrite groups suggest that their parent bodies accreted in three distinct regions. The parent bodies of ordinary and enstatite chondrites 
are inferred to have formed Sunward of the snow line whereas the CV, CI and $\mathrm{CM}$ carbonaceous chondrites formed in a water-rich region located beyond the snow line, perhaps within the orbits of the gas giant planets. In contrast, metal-rich chondrites apparently formed from a distinct ${ }^{26} \mathrm{Al}$-poor reservoir possibly located beyond the orbit of Saturn where cometary objects accreted. This proposal can be tested by investigating the $\mu^{54} \mathrm{Cr}-\mu^{26} \mathrm{Mg}$ * systematics of objects presumed to be of cometary origin such as interplanetary dust particles or ultra-carbonaceous micrometeorites (Busemann et al. 2009; Nesvorný et al. 2010; Duprat et al. 2010).

\subsection{Multiplicity of Chondrule-Forming Mechanisms}

Based on petrographic and mineralogical observations as well as chemical and oxygen isotopic compositions, it is thought that porphyritic chondrules formed by melting, typically incomplete, of isotopically diverse solid precursors in dustrich regions (dust/gas of $\sim 100-1000 \times$ solar) of the protoplanetary disk during repeatable and localised transient heating events (Krot and Nagashima 2017; Alexander et al. 2008; Cuzzi and Alexander 2006; Alexander and Ebel 2012). Among the proposed mechanisms of chondrule formation are shock waves related to disk gravitational instability, eccentric planetesimals, and X-ray flares (Desch et al. 2005; Morris et al. 2012), magnetised turbulence in the disk (McNally et al. 2013), collisions between chondritic or differentiated planetesimals (Asphaug et al. 2011; Johnson et al. 2015), and splashing of differentiated planetesimals (Asphaug et al. 2011). Although none of the proposed mechanisms can be completely ruled out, the common presence of relict grains and $\mathrm{Fe}, \mathrm{Ni}$-metal in porphyritic chondrules as well as the large age range of chondrules from individual chondrites, including ages indistinguishable from CAIs (Connelly et al. 2012; Bollard et al. 2017), are inconsistent with formation of the majority of porphyritic chondrules by splashing of differentiated bodies.

It is well accepted that the magnesian non-porphyritic chondrules in CB chondrites are thought to have formed in a melt-gas plume generated by a hypervelocity collision between planetesimals 4.8 Myr after CV CAIs (Bollard et al. 2015; Krot et al. 2005a), with one at least one of the colliding bodies being of differentiated nature (Fedkin et al. 2015; Oulton et al. 2016). In contrast to porphyritic chondrules, the $\mathrm{CB}$ chondrules formed during a single-stage event and, therefore, represent single generation objects, including the magnesian cryptocrystalline $\mathrm{CB}$ chondrules thought to have formed by condensation as melt droplets from the impact plume (Fedkin et al. 2015). The $\mathrm{CH}$ metal-rich carbonaceous chondrites, thought to be genetically related to CB chondrites (Weisberg et al. 1995; Krot et al. 2002), contain multiple generations of chondrules formed by different mechanisms. In detail, the magnesian non-porphyritic chondrules formed in the CB impact plume whereas the ferromagnesian and Al-rich porphyritic chondrules formed by incomplete melting of isotopically diverse precursors from multiple heating events unrelated to the $\mathrm{CB}$ impact (Krot et al. 2007; Krot et al. 2017). This clearly emphasises that chondrules formed by distinct mechanisms may occur in individual chondrite groups. In this 
case, however, the two formation mechanisms result in easily identifiable distinct petrologic features for chondrules. Accepting that shock waves are the dominant heat source for producing chondrules in most chondrite groups, this raises the possibility that the spectrum of petrologic features observed in chondrules is the expression of the numerous potential sources of shock waves that were active during the lifetime of the protoplanetary disk.

The proposal that the combined $\mu^{26} \mathrm{Mg}^{*}-\mu^{54} \mathrm{Cr}$ systematics of Solar System objects can be used to track their formation regions predicts that the chondruleforming process operated at various orbital distances, namely from the inner protoplanetary disk to beyond the accretion regions of the giant planets. Although the high surface densities and high energy environments typical of the inner protoplanetary disk permit chondrule formation through shock-related transient heating events (Connolly et al. 2006), thermal processing of solids in the outer Solar System may require a different source of shocks. For example, the energy required for the thermal processing of dust in the outer Solar System may result from planetary embryos bow shocks or, alternatively, impacts. This emphasises that the energy source required to melt dust resulting in the production of chondrules may be variable in both space and time during the evolution of the protoplanetary disk.

\subsection{Tracking Mass Transport and Recycling}

Similar to bulk asteroidal and planetary material, the stable isotopic compositions of individual chondrules allow us to determine the formation regions of their precursor material. Based on a limited dataset, Trinquier et al. (2009) identified correlated ${ }^{46} \mathrm{Ti}$ and ${ }^{50} \mathrm{Ti}$ nucleosynthetic variability in chondrules from the Allende CV3 chondrite, defining both excesses and deficits compared to the terrestrial composition. Moreover, Connelly et al. (2012) documented ${ }^{54} \mathrm{Cr}$ variability in individual Allende chondrules as well as chondrules from the NWA 5697 ordinary chondrite. At face value, these results indicate that chondrules formed from isotopically heterogeneous precursor material in different regions of the protoplanetary disk and were then transported to accretion regions of their respective parent bodies.

Accepting that the carbonaceous chondrites did indeed accrete beyond the snow line, a detailed investigation of the $\mu^{54} \mathrm{Cr}$ compositions of individual carbonaceous chondrite chondrules can provide insights into the transport of material to the accretion region(s) or carbonaceous chondrites. Using this approach, Olsen et al. (2016) recently reported $\mu^{54} \mathrm{Cr}$ data of 42 individual chondrules from $\mathrm{CV}$ and CR chondrites. Their analysis establishes that considerable $\mu^{54} \mathrm{Cr}$ variability exists amongst individual chondrules, most noticeably for CV chondrites (Fig. 6.10). In detail, $\mathrm{CV}$ chondrules record a range $\mu^{54} \mathrm{Cr}$ compositions that is comparable to that defined by our samples of chondrites, achondrites as well as meteorites from Mars and the Moon. Although it is possible that the $\mu^{54} \mathrm{Cr}$ heterogeneity observed in $\mathrm{CV}$ chondrules reflects the variable incorporation of a ${ }^{54} \mathrm{Cr}$ presolar carrier, this 

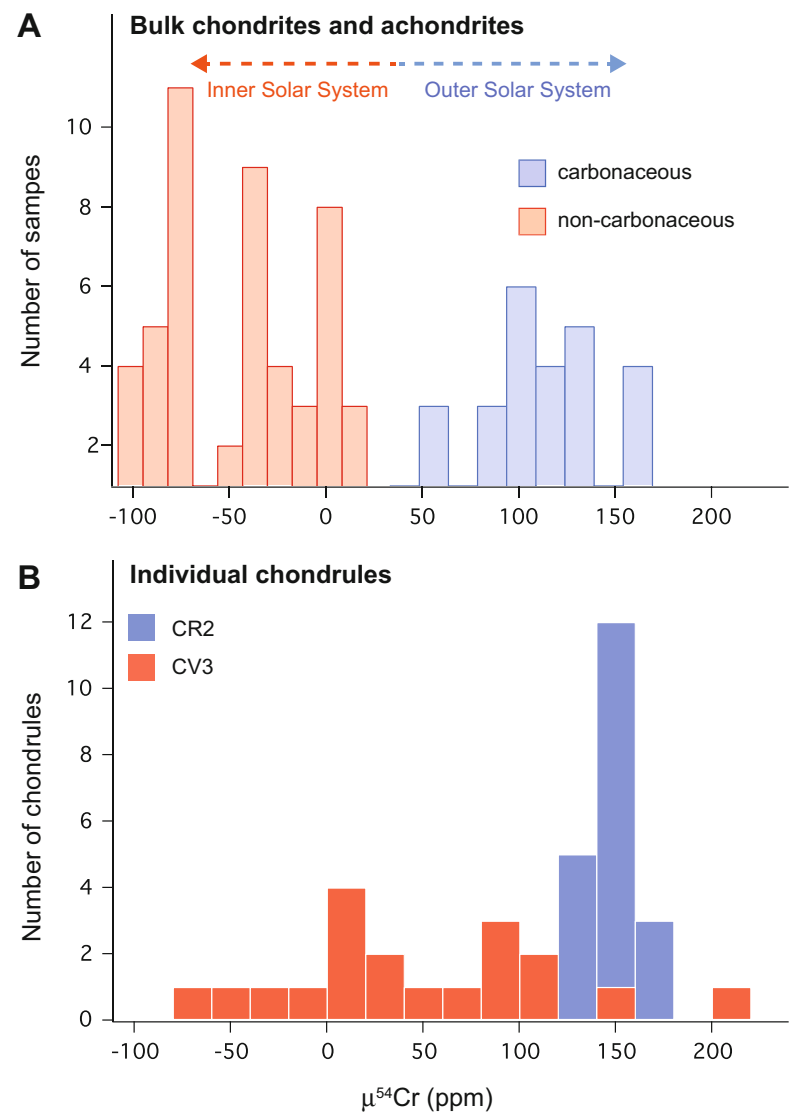

Fig. 6.10 $\mu^{54} \mathrm{Cr}$ variability amongst Solar System solids, asteroids and planetary bodies. (a) $\mu^{54} \mathrm{Cr}$ values for bulk chondrites and achondrites. The carbonaceous group comprises CI, CM, $\mathrm{CR}, \mathrm{CV}, \mathrm{CK}, \mathrm{CB}$ chondrites as well as lithic clasts from the Isheyevo $\mathrm{CH} / \mathrm{CB}$ chondrite. The non-carbonaceous group consists of ordinary and enstatite chondrites, angrites, aubrites, eucrites, diogenites, mesosiderites, acapulcoites and ureilites as well as Martian meteorites. The carbonaceous chondrites are thought to have accreted in a water-rich reservoir beyond the snow line whereas the non-carbonaceous chondrites and achondrites are believed to have formed Sunward of the snow line. (b) $\mu^{54} \mathrm{Cr}$ values for individual chondrules from $\mathrm{CV}$ and $\mathrm{CR}$ carbonaceous chondrites. The scale of the $x$-axis is the same for both panels. Data from Connelly et al. (2012), Van Kooten et al. (2016), Olsen et al. (2016), Larsen et al. (2011), Trinquier et al. (2007), Yamakawa et al. (2010), and Qin et al. (2010)

interpretation is not consistent with the observed range of compositions. Indeed, given that the scale at which individual chondrules sample disk material is at least 3 orders of magnitude smaller relative to the size of the samples typically used to define the ${ }^{54} \mathrm{Cr}$ composition of chondrites and achondrites, greater variability is predicted to be recorded by individual chondrules. The comparable range of ${ }^{54} \mathrm{Cr}$ compositions observed for chondrules and bulk samples of chondrites and 
achondrites points to a similar process imparting the ${ }^{54} \mathrm{Cr}$ variation, namely thermal processing of their precursor material. If correct, this establishes that the ${ }^{54} \mathrm{Cr}$ values of early Solar System materials can be used to provide a high fidelity record of the formation region of their precursor material.

The range of $\mu^{54} \mathrm{Cr}$ compositions recorded by $\mathrm{CV}$ chondrite chondrules, with both excesses and deficits relative to the terrestrial composition, requires that these objects formed from precursors that originated at various orbital distances, namely from the accretion regions of most differentiated asteroids and terrestrial planets to the formation regions of carbonaceous chondrites. In accord with the abundance of refractory inclusions formed close to the young Sun in CV chondrites (Krot et al. 2009), the occurrence of chondrules with inner Solar System $\mu^{54} \mathrm{Cr}$ signatures in these chondrites requires efficient lateral outward transport of material during the earliest stages of the evolution of the solar protoplanetary disk. In contrast to $\mathrm{CV}$ chondrules, $\mathrm{CR}$ chondrules define a much more restricted range of $\mu^{54} \mathrm{Cr}$ values characterised by compositions found in bulk carbonaceous chondrites. This observation suggests that CR chondrules formed from precursors predominantly formed in the accretion region of their parent body, with little input of material with inner Solar System $\mu^{54} \mathrm{Cr}$ signatures. This is consistent with the low abundance of CAIs in CR chondrites thereby supporting the view of limited transport of inner Solar System solids to their accretion region. Collectively, the $\mu^{54} \mathrm{Cr}$ systematics of individual chondrules suggest that variable recycling of thermally processed inner disk solids to the accretion of carbonaceous chondrites may be an important process regulating the compositions of the various carbonaceous chondrite groups.

The coupled $\mu^{54} \mathrm{Cr}$ and $\mu^{26} \mathrm{Mg} *$ data for individual chondrules from $\mathrm{CV}$ and $\mathrm{CR}$ chondrites support the idea that the accretion region of metal-rich chondrites and their components must have been isolated from that of $\mathrm{CV}$ chondrites (Van Kooten et al. 2016; Olsen et al. 2016). Indeed, chondrules from CV chondrites show broadly correlated $\mu^{54} \mathrm{Cr}-\mu^{26} \mathrm{Mg}$ * variability, similarly to bulk inner Solar System reservoirs (Fig. 6.9). The lack of evidence for admixing of appreciable amounts of thermally unprocessed primordial molecular cloud material in the precursors of CV chondrules suggest that their accretion region(s) was spatially isolated from that of metal-rich chondrites. In other words, inward transport of outer Solar System millimetre-sized solids to the accretion region of $\mathrm{CV}$ chondrites appears to have been limited for a significant period of the disk lifetime. A possible mechanism to limit the inward migration of outer Solar System material is the formation of gas giants opening gaps in the protoplanetary disk (Müller and Kley 2013), providing that the gas giants are able to accrete most of the solids attempting to cross the gap. Recent numerical simulations show that rapid formation of gas and ice giants can occur by accretion of centimetre-to-metre-sized particles by the mechanism of pebble accretion (Lambrechts and Johansen 2012). In these models, pebbles are concentrated by aerodynamic drag and gravitationally collapse to form objects of up to $1000 \mathrm{~km}$ in diameter; these planetary embryos can then efficiently accrete leftover pebbles and directly form the cores of giant planets. Therefore, the growth of giant cores by pebble accretion provides an efficient means of limiting the influx 
of material by generating partial disk gaps and pressure bumps outside of their orbits (Lambrechts et al. 2014).

\subsection{Outward Mass Transport Mechanisms}

The $\mu^{54} \mathrm{Cr}$ data of individual chondrules from $\mathrm{CV}$ and $\mathrm{CR}$ chondrites suggest variable amounts of transport and recycling of inner Solar System material to the accretion regions of their respective parent bodies. Two classes of models have been invoked to explain how high temperature refractory material was redistributed throughout the disk to be incorporated into primitive bodies. One class of models are disk models, which explore how the inward transport of mass and angular momentum may result in outward transport in the early evolution of protoplanetary disks. For example, it has been suggested that in viscously evolving disks, turbulence can combine with the large-scale flows of the disk to carry refractory material outward against the inward motions associated with gas drag and accretion thereby providing a means to preserve and diffuse material at larger orbital distances (Cuzzi et al. 2003; Ciesla 2007, 2010). In these models, the transport of material is apparently most efficient in highly turbulent disks, which may limit the efficiency of this mechanism to the earliest stages. Recent studies suggest that the accretion of differentiated planetesimals was initiated within a few $10^{5}$ years of CAI formation (Schiller et al. 2011; Kruijer et al. 2014; Schiller et al. 2015a; Larsen et al. 2016). Thus, a consequence of outward diffusion via the disk midplane is the incorporation of refractory material within early formed asteroidal and planetary embryos. However, the coupled ${ }^{54} \mathrm{Cr}$ and ${ }^{50} \mathrm{Ti}$ systematics of Earth, Mars, most differentiated asteroids as well as ordinary and enstatite chondrites (Trinquier et al. 2009) suggest a paucity of CAI material in their precursors, implying that outward diffusive transport through the midplane may not have been the dominant transport mechanism.

The second type of outward transport models is based on the magnetically driven outflows characteristic of young stellar objects. Both observations and simulations show that protostars exhibit powerful outflows of material accelerated to supersonic speeds along the polar axis of the star or as winds from the disk (Bontemps et al. 1996; Romanova et al. 2009; Sheikhnezami et al. 2012). These outflows provide an efficient mechanism for releasing the angular momentum inherited from the accretion process. Solids may be entrained and accelerated in jets and winds resulting in the outward transport of material in ballistic trajectories above the disk (Shu et al. 1996; Hu 2010). Although the role of stellar outflows in the formation of chondritic components such as CAIs and chondrules is uncertain (Desch et al. 2010), disk winds and jets are generic features of protostars and, thus, can provide a potentially efficient mechanism for outward transport and recycling of material during the entire lifetime of the protoplanetary disk (Hansen 2014).

The paucity of CAIs as well as the lack of chondrules with an inner solar system signature in the accretion region of the CR chondrite parent body, inferred to have been beyond the orbits of the gas giants (Van Kooten et al. 2016), suggested little 
input of thermally processed inner Solar System solids to the outer solar system. Thus, outward mass transport to large orbital distances such as the accretion region of cometary objects may have been only possible during the early, deeply embedded stage of the proto-Sun characterised by powerful high-velocity jets (Bontemps et al. 1996). In contrast, approximately 50\% of the chondrules in CV3 chondrites have $\mu^{54} \mathrm{Cr}$ compositions typical of inner Solar System solids indicating significant admixing of inner Solar System material to the accretion region of the CV parent body. The high abundance of CAI material in CV chondrites relative to CR supports this observation. Thus, outward transport and recycling of inner Solar System material to the outer part of the asteroid belt may have been more protracted and, hence, driven by lower-velocity stellar and disk winds, which may have been active for the entire duration of the accretion phase of the proto-Sun (Reipurth and Bally 2001).

\subsection{The Chondrule-Matrix Complementarity}

The bulk chemistry of chondrites is defined by the two major components, chondrules and matrix. A number of studies have investigated the apparent chemical relationship between chondrules and matrix in individual chondrite groups (Hezel and Palme 2010; Palme et al. 2015; Ebel et al. 2016). These studies have concluded that the average compositions of chondrules and matrix are typically different for a number of elements in an individual chondrite whereas the bulk composition, which reflects a mixtures of chondrules and matrix, has approximately a solar elemental abundance. This so-called chondrule-matrix complementary has been used to argue for a genetic link between these two components and, therefore, formation from a single reservoir. Given the short residence time of solids in a protoplanetary disk due to gas drag (Weidenschilling 1977), this model, in its simplest expression, predicts that all chondrules from a single chondrite should be in isotopic equilibrium and have the same age. Thus, the chondrule-matrix complementarity requires that chondrule formation and asteroidal accretion are intimately linked. However, the observed $\mu^{54} \mathrm{Cr}$ variability suggests that chondrules from individual chondrite groups formed from isotopically diverse precursor material in different regions of the protoplanetary disk and were subsequently transported to the accretion regions of their respective parent bodies. This is consistent with the presence of age variability of $\sim 3 \mathrm{Myr}$ between chondrules from individual chondrites (Connelly et al. 2012), which requires transport and/or storage. At face value, these data appear inconsistent with the concept of chondrule-matrix complementary as originally envisaged, namely that all chondrules from an individual chondrites are all genetically related to the coexisting matrix.

Recent models of evolving viscous disks, however, suggest that a complementary relationship between chondrules and dust can be preserved for long time-scales provided that the decoupling between chondrules and gas is limited (Goldberg et al. 2015). In these models, various chondrule populations remained in complementarity 
such that the bulk contribution from each source is chemically solar and, thus, so is the final mixture. However, these experiments assume that the main transport mechanism of chondrules occurs through outward diffusion via the disk midplane. In disk models where outward transport of material is associated with stellar outflows (Shu et al. 1996; Hu 2010), the coarse-grained dust component (i.e. CAIs and chondrules) is not expected to be efficiently coupled to the gas and, thus, it is unclear how complementary can be preserved. A possibility is the observed chondrule-matrix complementarity is an expression of the generic process of chondrule formation and does not reflect a genetic link. In this view, the matrix comprises a complement related to the chondrule formation process (Alexander 2005) such that the bulk composition of the matrix is shifted from its starting composition and, thus, appears complementary to a chondrule composition. This does not require that the matrix is genetically linked to the chondrules in an individual chondrite but merely that some of it has experienced earlier chondrule formation events. In this view, fractions of the matrix in a particular chondrite may be complementary to chondrule populations in other chondritic meteorites.

\subsection{Summary and Perspectives}

The U-corrected $\mathrm{Pb}-\mathrm{Pb}$ ages of individual CAIs and chondrules provide a robust framework to understand the chronology of solid formation in the early Solar System. CAIs formed during a brief time interval of less than $\sim 0.2 \mathrm{Myr}$, possibly associated with the early stages of the proto-Sun characterised by high mass accretion rates. In contrast, the production of chondrules began contemporaneously with the formation of canonical CAIs and lasted for the entire lifetime of the solar protoplanetary disk (Fig. 6.11). All chondrite groups investigated contain chondrules of multiple generations with a similar age range of $~ 3 \mathrm{Myr}$. The bulk compositions of different chondrite parent bodies (CI, CM, CV, CO, OC and EC) record significant stable isotope heterogeneity of nucleosynthetic origin for various elements, which is interpreted as reflecting the selective destruction of isotopically anomalous presolar carriers during progressive thermal processing in the inner disk region. This nucleosynthetic variability indicates that these chondrite parent bodies accreted in spatially distinct disk regions. The level of this heterogeneity is comparable to the range of nucleosynthetic variability among individual chondrules from CV chondrites. Thus, CV chondrules and/or their precursors originated in different parts of the protoplanetary disk and were subsequently transported to the accretion region of the $\mathrm{CV}$ parent asteroid. Combined with the protracted formation timescales inferred by the absolute $\mathrm{U}$-corrected $\mathrm{Pb}-\mathrm{Pb}$ dating method, the observed variability in the stable isotope compositions of chondrules paints a dynamic picture of the early Solar System, where chondrule formation, transport and recycling occur continuously during the lifetime of the protoplanetary disk. Thus, accretion of individual chondrite parent bodies was a continuous process. Given the stable isotope heterogeneity recorded by individual chondrules, the 


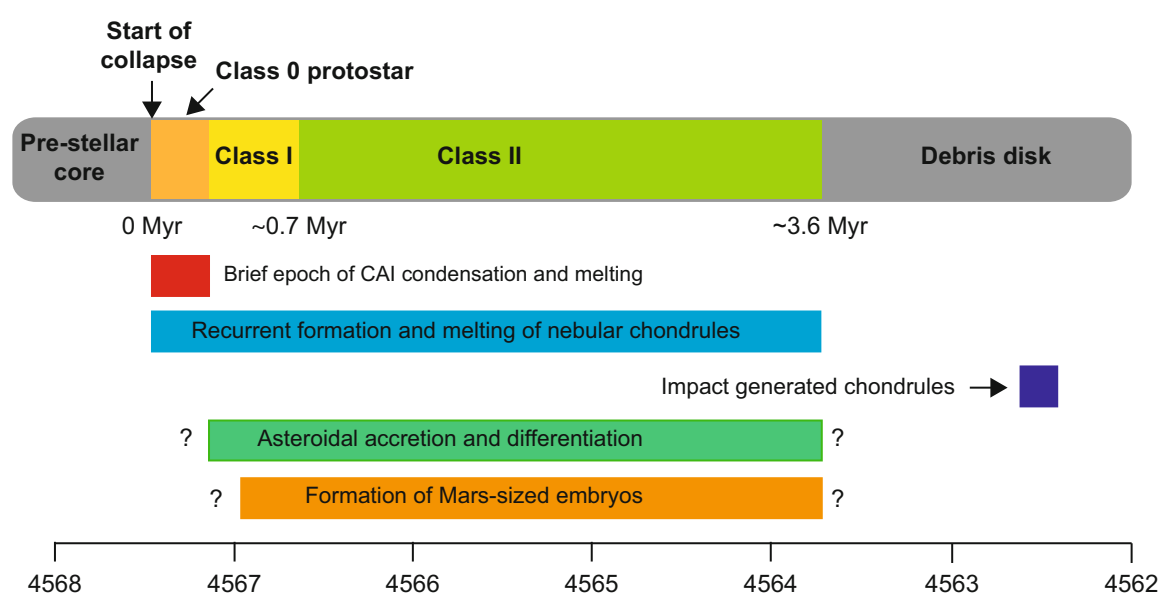

Absolute ages (Myr)

Fig. 6.11 Time scales of solid formation and disk evolution. The brief formation interval for the formation of CAIs is similar to the median lifetimes of class 0 protostars of 0.1 to $0.2 \mathrm{Myr}$ inferred from astronomical observations. Therefore, the thermal regime required for CAI condensation may only have existed during the earliest stages of disk evolution typified by high mass accretion rates onto the central star. In contrast, recurrent chondrule formation occurred throughout the lifetime of the protoplanetary disk. The accretion and differentiation of asteroidal bodies, possibly leading to the growth of Mars-sized planetary embryos within $\sim 4-5 \mathrm{Myr}$, may have been promoted by chondrule accretion (Johansen et al. 2015). Modified from Connelly et al. (2012)

inferred continuous, layered accretion of chondrite parent bodies predicts the existence of time-dependent isotope heterogeneity within these bodies. The growth of sizeable asteroidal bodies and planetary embryos occurred during the lifetime of the protoplanetary disk, possibly promoted by the gas-drag assisted accretion of chondrules onto asteroidal seeds.

The metal-rich chondrites, namely the $\mathrm{CR}, \mathrm{CH}$ and $\mathrm{CB}$ chondrites, formed from precursor material that has largely escaped the thermal processing recorded by the inner Solar System bodies. The metal-rich chondrites and their chondrules appear to have incorporated appreciable amounts of thermally unprocessed primordial molecular cloud material, suggesting formation in the outer part of the Solar System, beyond the orbits the gas giant planets. Thus, thermal processing of solids, including chondrule formation was not restricted to the inner disk regions but also occurred in the outer Solar System. However, the mechanism and efficiency of the thermal processing of solids at large orbital distances are poorly understood.

The stable isotope compositions of the inner and outer Solar System materials are distinct, implying limited mixing of these two reservoirs. An efficient mechanism to limit the inward transport of outer disk solids to the inner Solar System is the formation of gas giants opening gaps in the disk. Collectively, these data suggest that different chondrule-forming mechanisms may have operated at distinct times and/or regions of the protoplanetary disk. Indeed, $\mathrm{CH}$ chondrites contain 
chondrules formed by different mechanisms, including gas-melt impact plume produced chondrules as well as chondrules formed by incomplete melting during transient heating events.

Outward transport of solids could have occurred by a variety of time-dependent processes, including turbulent diffusion and stellar outflows. The outward mass transport to large orbital distances such as the accretion region of cometary objects may have been only possible during the early, deeply embedded stage of the proto-Sun characterised by powerful high-velocity jets. The paucity of CAIs in the accretion regions of inner protoplanetary disk bodies relative to the accretion regions of carbonaceous chondrites and the presence of CAI-like objects in the Jupiter family comet $81 \mathrm{P} /$ Wild 2 suggest that refractory inclusions were radially transported above the midplane, possibly entrained and accelerated in stellar outflows such as jets and disk winds. In contrast, outward transport and recycling of inner Solar System material to the outer part of the asteroid belt appear to be more protracted and, hence, driven by lower-velocity stellar and disk winds, which may have been active for the entire duration of the accretion phase of the proto-Sun.

The scenario presented here for the protoplantary disk evolution can be tested through key isotope measurements of various meteoritic solids. The continuous layered accretion of chondrite parent bodies can be tested by high-precision bulk $\mathrm{Mg}$ and $\mathrm{Cr}$ isotope measurements of chondrites thought to have originated at different depths of the same parent asteroid such as ordinary chondrites of petrologic types 3-6 that experienced increasingly higher peak metamorphic temperatures (300-900 $\left.{ }^{\circ} \mathrm{C}\right)$. A better understanding of the timing and tempo of chondrule formation and, by extension, the nature of the chondrule forming mechanisms that may have operated at different times can be evaluated by acquiring a statistically significant dataset of $\mathrm{U}$-corrected $\mathrm{Pb}-\mathrm{Pb}$ ages of individual chondrules from various chondrite groups. The proposal of a reduced inner Solar System initial abundance of ${ }^{26} \mathrm{Al}$ relative to the canonical ratio observed in CAIs can be tested by combined $\mathrm{Al}-\mathrm{Mg}$ and $\mathrm{U}$-corrected $\mathrm{Pb}-\mathrm{Pb}$ ages of individual chondrules from primitive chondrites. The hypothesis that metal-rich chondrites accreted in an ${ }^{26} \mathrm{Al}$-poor region of the outer Solar System can be verified by investigating the $\mu^{54} \mathrm{Cr}-\mu^{26} \mathrm{Mg} *$ systematics of objects presumed to be of cometary origin such as interplanetary dust particles or ultra-carbonaceous micrometeorites. Finally, the roles of stellar outflows in the outward transport of solids can be evaluated by both high-resolution astronomical observations of young disks and realistic numerical simulations of star-formation and evolving protoplanetary disks.

Acknowledgements This work was funded by grants from the Danish National Research Foundation (DNRF97) and from the European Research Council (ERC Consolidator Grant Agreement 616027-STARDUST2ASTEROIDS) to MB. We thank the referee for comments, which have improved our chapter. 


\section{References}

Abreu, N.M., Brearley, A.J.: Early solar system processes recorded in the matrices of two highly pristine CR3 carbonaceous chondrites, MET 00426 and QUE 99177. Geochim. Cosmochim. Acta 74, 1146-1171 (2010). doi:10.1016/j.gca.2009.11.009

Alexander, C.M.O.D.: Re-examining the role of chondrules in producing the elemental fractionations in chondrites. Meteorit. Planet. Sci. 40, 943 (2005). doi:10.1111/j.19455100.2005.tb00166.x

Alexander, C.M.O.D., Ebel, D.S.: Questions, questions: Can the contradictions between the petrologic, isotopic, thermodynamic, and astrophysical constraints on chondrule formation be resolved? Meteorit. Planet. Sci. 47, 1157-1175 (2012). doi:10.1111/j.1945-5100.2011.01308.x

Alexander, C.O., et al.: The formation conditions of chondrules and chondrites. Science 320, 1617 (2008). doi:10.1126/science.1156561. http://adsabs.harvard.edu/abs/2008Sci...320.1617A

Amelin, Y., Krot, A.: Pb isotopic age of the Allende chondrules. Meteorit. Planet. Sci. 42:13211335 (2007). doi:10.1111/j.1945-5100.2007.tb00577.x

Amelin, Y., Krot, A.N., Hutcheon, I.D., Ulyanov, A.A.: Lead isotopic ages of chondrules and calcium-aluminum-rich inclusions. Science 297, 1678-1683 (2002). doi: $10.1126 /$ science. 1073950

Asphaug, E., Jutzi, M., Movshovitz, N.: Chondrule formation during planetesimal accretion. Earth Planet. Sci. Lett. 308, 369-379 (2011). doi:10.1016/j.eps1.2011.06.007

Becker, M., Hezel, D.C., Schulz, T., Elfers, B.M., Münker, C.: Formation timescales of CV chondrites from component specific Hf-W systematics. Earth Planet. Sci. Lett. 432, 472-482 (2015). doi:10.1016/j.epsl.2015.09.049

Birck, J.L.: An overview of isotopic anomalies in extraterrestrial materials and their nucleosynthetic heritage. Rev. Mineral. Geochem. 55(1), 25-64 (2004). doi:10.2138/gsrmg.55.1.25. http://rimg.geoscienceworld.org/content/55/1/25

Bitsch, B., Lambrechts, M., Johansen, A.: The growth of planets by pebble accretion in evolving protoplanetary discs. Astron. Astrophys. 582, A112 (2015). doi:10.1051/00046361/201526463, 1507.05209

Bizzarro, M., et al.: High-precision mg-isotope measurements of terrestrial and extraterrestrial material by HR-MC-ICPMS - implications for the relative and absolute mg-isotope composition of the bulk silicate earth. J. Anal. At. Spectrom. 26, 565 (2011). doi:10.1039/C0JA00190B. http://pubs.rsc.org/en/content/articlelanding/2011/ja/c0ja00190b

Bizzarro, M., Olsen, M., Itoh, S., Kawasaki, N., Schiller, M., Bonal, L., Yurimoto, H.: Evidence for a reduced initial abundance of ${ }^{26} \mathrm{Al}$ in chondrule forming regions and implications for the accretion timescales of protoplanets. In: 77th Annual Meeting of the Meteoritical Society, LPI Contributions, vol. 1800, p. 5125 (2014)

Bollard, J., Connelly, J.N., Bizzarro, M.: Pb-Pb dating of individual chondrules from the $\mathrm{CB}_{a}$ chondrite Gujba: assessment of the impact plume formation model. Meteorit. Planet. Sci. 50, 1197-1216 (2015). doi:10.1111/maps.12461

Bollard, J., Connelly, J.N., Whitehouse, M.J., Pringle, E.A., Bonal, L., Jørgensen, J.K., Nordlund, Å., Moynier, F., Bizzarro, M.: Early formation of planetary building blocks inferred from $\mathrm{Pb}$ isotopic ages of chondrules. Sci. Adv. 3, e1700407 (2017)

Bontemps, S., Andre, P., Terebey, S., Cabrit, S.: Evolution of outflow activity around low-mass embedded young stellar objects. Astron. Astrophys. 311, 858-872 (1996)

Boss, A.P., Graham, J.A.: Clumpy disk accretion and chondrule formation. Icarus 106, 168 (1993). doi:10.1006/icar.1993.1164

Brennecka, G.A., Weyer, S., Wadhwa, M., Janney, P.E., Zipfel, J., Anbar, A.D.: ${ }^{238} \mathrm{U} /{ }^{235} \mathrm{U}$ variations in meteorites: extant ${ }^{247} \mathrm{Cm}$ and implications for $\mathrm{Pb}-\mathrm{Pb}$ dating. Science 327, 449 (2010). doi:10.1126/science.1180871

Brennecka, G.A., Budde, G., Kleine, T.: Uranium isotopic composition and absolute ages of Allende chondrules. Meteorit. Planet. Sci. 50, 1995 (2015). doi:10.1111/maps.12567 
Budde, G., Kleine, T., Kruijer, T.S., Burkhardt, C., Metzler, K.: Tungsten isotopic constraints on the age and origin of chondrules. Proc. Natl. Acad. Sci. USA 113(11), 2886-2891 (2016). doi:10.1073/pnas.1524980113. http://www.pnas.org/content/113/11/2886

Busemann, H., Nguyen, A.N., Cody, G.D., Hoppe, P., Kilcoyne, A.L.D., Stroud, R.M., Zega, T.J., Nittler, L.R.: Ultra-primitive interplanetary dust particles from the comet 26P/Grigg-Skjellerup dust stream collection. Earth Planet. Sci. Lett. 288, $44-57$ (2009). doi:10.1016/j.epsl.2009.09.007

Carporzen, L., Weiss, B.P., Elkins-Tanton, L.T., Shuster, D.L., Ebel, D., Gattacceca, J.: Magnetic evidence for a partially differentiated carbonaceous chondrite parent body. Proc. Natl. Acad. Sci. USA 108(16), 6386-6389 (2011). doi:10.1073/pnas.1017165108. http://www.pnas.org/ content/108/16/6386

Ciesla, F.J.: Outward transport of high-temperature materials around the midplane of the solar nebula. Science 318, 613 (2007). doi:10.1126/science.1147273. http://adsabs.harvard.edu/abs/ 2007Sci...318..613C

Ciesla, F.J.: The distributions and ages of refractory objects in the solar nebula. Icarus 208, 455 467 (2010). doi:10.1016/j.icarus.2010.02.010

Connelly, J.N., Bizzarro, M.: $\mathrm{Pb}-\mathrm{Pb}$ dating of chondrules from $\mathrm{CV}$ chondrites by progressive dissolution. Chem. Geol. 259(3-4), 143-151 (2009). doi:10.1016/j.chemgeo.2008.11.003. http://www.sciencedirect.com/science/article/pii/S0009254108005081

Connelly, J.N., Amelin, Y., Krot, A.N., Bizzarro, M.: Chronology of the solar system's oldest solids. Astrophys. J. Lett. 675, L121 (2008). doi:10.1086/533586

Connelly, J.N., Bizzarro, M., Krot, A.N., Nordlund, A., Wielandt, D., Ivanova, M.A.: The absolute chronology and thermal processing of solids in the solar protoplanetary disk. Science 338, 651 (2012). doi:10.1126/science.1226919. http://adsabs.harvard.edu/abs/2012Sci...338..651C

Connolly, H.C. Jr., Love, S.G.: The formation of chondrules: petrologic tests of the shock wave model. Science 280, 62-67 (1998). doi:10.1126/science.280.5360.62

Connolly, H.C. Jr., Desch, S.J., Ash, R.D., Jones, R.H.: Transient heating events in the protoplanetary nebula. In: Lauretta, D.S., McSween, H.Y. (eds.) Meteorites and the Early Solar System II, pp. 383-397. University of Arizona Press, Tucson (2006)

Cuzzi, J.N., Alexander, C.M.O.D.: Chondrule formation in particle-rich nebular regions at least hundreds of kilometres across. Nature 441, 483-485 (2006). doi:10.1038/nature04834

Cuzzi, J.N., Davis, S.S., Dobrovolskis, A.R.: Blowing in the wind. II. Creation and redistribution of refractory inclusions in a turbulent protoplanetary nebula. Icarus 166, 385-402 (2003). doi:10.1016/j.icarus.2003.08.016

Dauphas, N., Pourmand, A.: Hf-W-Th evidence for rapid growth of Mars and its status as a planetary embryo. Nature 473, 489-492 (2011). doi:10.1038/nature10077

Desch, S.J., Ciesla, F.J., Hood, L.L., Nakamoto, T.: Heating of chondritic materials in solar nebula shocks. In: Krot, A.N., Scott, E.R.D., Reipurth, B. (eds.) Chondrites and the Protoplanetary Disk. Astronomical Society of the Pacific Conference Series, vol. 341, p. 849. Astronomical Society of the Pacific, San Francisco (2005)

Desch, S.J., Morris, M.A., Connolly, H.C. Jr., Boss, A.P.: A critical examination of the X-wind model for chondrule and calcium-rich, aluminum-rich inclusion formation and radionuclide production. Astrophys. J. 725, 692-711 (2010). doi:10.1088/0004-637X/725/1/692, 1011.3483

Duprat, J., Dobrica, E., Engrand, C., Aléon, J., Marrocchi, Y., Mostefaoui, S., Meibom, A., Leroux, H., Rouzaud, J.N., Gounelle, M., Robert, F.: Extreme deuterium excesses in ultracarbonaceous micrometeorites from central antarctic snow. Science 328, 742-745 (2010). doi:10.1126/science.1184832. http://science.sciencemag.org/content/328/5979/742

Ebel, D.S., Brunner, C., Konrad, K., Leftwich, K., Erb, I., Lu, M., Rodriguez, H., Crapster-Pregont, E.J., Friedrich, J.M., Weisberg, M.K.: Abundance, major element composition and size of components and matrix in CV, CO and Acfer 094 chondrites. Geochim. Cosmochim. Acta 172, 322-356 (2016). doi:10.1016/j.gca.2015.10.007

Evans, N.J. II, Dunham, M.M., Jørgensen, J.K., Enoch, M.L., Merín, B., van Dishoeck, E.F., Alcalá, J.M., Myers, P.C., Stapelfeldt, K.R., Huard, T.L., Allen, L.E., Harvey, P.M., van Kempen, T., Blake, G.A., Koerner, D.W., Mundy, L.G., Padgett, D.L., Sargent, A.I.: The spitzer 
c2d legacy results: star-formation rates and efficiencies; evolution and lifetimes. Astrophys. J. Suppl. 181, 321 (2009). doi:10.1088/0067-0049/181/2/321. http://adsabs.harvard.edu/abs/ 2009ApJS..181..321E

Fedkin, A.V., Grossman, L., Humayun, M., Simon, S.B., Campbell, A.J.: Condensates from vapor made by impacts between metal-, silicate-rich bodies: comparison with metal and chondrules in CB chondrites. Geochim. Cosmochim. Acta 164, 236-261 (2015). doi:10.1016/j.gca.2015.05.022

Goldberg, A.Z., Owen, J.E., Jacquet, E.: Chondrule transport in protoplanetary discs. Mon. Not. R. Astron. Soc. 452, 4054-4069 (2015). doi:10.1093/mnras/stv1610

Greshake, A.: The primitive matrix components of the unique carbonaceous chondrite ACFER 094: a TEM study. Geochim. Cosmochim. Acta 61, 437-452 (1997). doi:10.1016/S00167037(96)00332-8

Grossman, J.N., Rubin, A.E., Nagahara, H., King, E.A.: Properties of chondrules. In: Kerridge, J.F., Matthews, M.S. (eds.) Meteorites and the Early Solar System, pp. 619-659. University of Arizona Press, Tucson (1988)

Hansen, B.M.S.: The circulation of dust in protoplanetary discs and the initial conditions of planet formation. Mon. Not. R. Astron. Soc. 440, 3545-3556 (2014). doi:10.1093/mnras/stu471, 1403.6552

Hezel, D.C., Palme, H.: The chemical relationship between chondrules and matrix and the chondrule matrix complementarity. Earth Planet. Sci. Lett. 294, 85-93 (2010). doi:10.1016/j.epsl.2010.03.008

Holst, J.C., Olsen, M.B., Paton, C., Nagashima, K., Schiller, M., Wielandt, D., Larsen, K.K., Connelly, J.N., Jørgensen, J.K., Krot, A.N., Nordlund, A., Bizzarro, M.: ${ }^{182} \mathrm{Hf}^{-182} \mathrm{~W}$ age dating of a ${ }^{26} \mathrm{Al}$-poor inclusion and implications for the origin of short-lived radioisotopes in the early Solar System. Proc. Natl. Acad. Sci. USA 110(22), 8819-8823 (2013). doi:10.1073/pnas.1300383110. http://www.pnas.org/content/110/22/8819

Hood, L.L.: Thermal processing of chondrule and CAI precursors in planetesimal bow shocks. Meteorit. Planet. Sci. 33 (1998). doi:10.1111/j.1945-5100.1998.tb01611.x

Hu, R.: Transport of the first rocks of the solar system by X-winds. Astrophys. J. 725, 1421-1428 (2010). doi:10.1088/0004-637X/725/2/1421, 1010.2532

Hutcheon, I.D., Hutchison, R.: Evidence from the Semarkona ordinary chondrite for Al-26 heating of small planets. Nature 337, 238-241 (1989). doi:10.1038/337238a0

Hutson, M., Ruzicka, A.: A multi-step model for the origin of E3 (enstatite) chondrites. Meteorit. Planet. Sci. 35, 601-608 (2000). doi:10.1111/j.1945-5100.2000.tb01440.x

Jacobsen, B., Yin, Q.Z., Moynier, F., Amelin, Y., Krot, A.N., Nagashima, K., Hutcheon, I.D., Palme, H.: ${ }^{26} \mathrm{Al}-{ }^{26} \mathrm{Mg}$ and ${ }^{207} \mathrm{~Pb}-{ }^{206} \mathrm{~Pb}$ systematics of Allende CAIs: canonical solar initial ${ }^{26} \mathrm{Al} /{ }^{27} \mathrm{Al}$ ratio reinstated. Earth Planet. Sci. Lett. 272(1-2), 353364 (2008). doi:10.1016/j.eps1.2008.05.003. http://www.sciencedirect.com/science/article/pii/ S0012821X0800318X

Johansen, A., Oishi, J.S., Mac Low, M.M., Klahr, H., Henning, T., Youdin, A.: Rapid planetesimal formation in turbulent circumstellar disks. Nature 448, 1022-1025 (2007). doi:10.1038/nature06086, 0708.3890

Johansen, A., Mac Low, M.M., Lacerda, P., Bizzarro, M.: Growth of asteroids, planetary embryos, and Kuiper belt objects by chondrule accretion. Sci. Adv. 1, 1500109 (2015). doi:10.1126/sciadv.1500109, 1503.07347

Johnson, B.C., Minton, D.A., Melosh, H.J., Zuber, M.T.: Impact jetting as the origin of chondrules. Nature 517, 339-341 (2015). doi:10.1038/nature14105

Jones, R.H., Leshin, L.A., Guan, Y., Sharp, Z.D., Durakiewicz, T., Schilk, A.J.: Oxygen isotope heterogeneity in chondrules from the Mokoia CV3 carbonaceous chondrite. Geochim. Cosmochim. Acta 68, 3423-3438 (2004). doi:10.1016/j.gca.2004.01.013

Joung, M.K.R., Mac Low, M.M., Ebel, D.S.: Chondrule formation and protoplanetary disk heating by current sheets in nonideal magnetohydrodynamic turbulence. Astrophys. J. 606, 532-541 (2004). doi:10.1086/381651, astro-ph/0309189 
Kerridge, J.F.: Carbon, hydrogen and nitrogen in carbonaceous chondrites Abundances and isotopic compositions in bulk samples. Geochim. Cosmochim. Acta 49, 1707-1714 (1985). doi:10.1016/0016-7037(85)90141-3

Kita, N.T., Ushikubo, T.: Evolution of protoplanetary disk inferred from ${ }^{26} \mathrm{Al}$ chronology of individual chondrules. Meteorit. Planet. Sci. 47, 1108-1119 (2012). doi:10.1111/j.19455100.2011.01264.x

Kita, N.T., Nagahara, H., Togashi, S., Morishita, Y.: A short duration of chondrule formation in the solar nebula: evidence from ${ }^{26} \mathrm{Al}$ in Semarkona ferromagnesian chondrules. Geochim. Cosmochim. Acta 64, 3913-3922 (2000). doi:10.1016/S0016-7037(00)00488-9

Kita, N.T., Nagahara, H., Tachibana, S., Tomomura, S., Spicuzza, M.J., Fournelle, J.H., Valley, J.W.: High precision SIMS oxygen three isotope study of chondrules in LL3 chondrites: role of ambient gas during chondrule formation. Geochim. Cosmochim. Acta 74, 6610-6635 (2010). doi:10.1016/j.gca.2010.08.011

Kita, N.T., Yin, Q.Z., MacPherson, G.J., Ushikubo, T., Jacobsen, B., Nagashima, K., Kurahashi, E., Krot, A.N., Jacobsen, S.B.: ${ }^{26} \mathrm{Al}-{ }^{26} \mathrm{Mg}$ isotope systematics of the first solids in the early solar system. Meteorit. Planet. Sci. 48, 1383-1400 (2013). doi:10.1111/maps.12141

Kleine, T., Touboul, M., Bourdon, B., Nimmo, F., Mezger, K., Palme, H., Jacobsen, S.B., Yin, Q.Z., Halliday, A.N.: Hf-W chronology of the accretion and early evolution of asteroids and terrestrial planets. Geochim. Cosmochim. Acta 73, 5150-5188 (2009). doi:10.1016/j.gca.2008.11.047

Krot, A.N., Nagashima, K.: Constraints on mechanisms of chondrule formation from chondrule precursors and chronology of transient heating events in the protoplanetary disk. Geochem. J. 51, 45-58 (2017)

Krot, A.N., Wasson, J.T.: Igneous rims on low-FeO and high-FeO chondrules in ordinary chondrites. Geochim. Cosmochim. Acta 59, 4951-4966 (1995). doi:10.1016/0016-7037(95) 00337-1

Krot, A.N., Meibom, A., Weisberg, M.K., Keil, K.: Invited review: the CR chondrite clan: implications for early solar system processes. Meteorit. Planet. Sci. 37, 1451-1490 (2002). doi:10.1111/j.1945-5100.2002.tb00805.x

Krot, A.N., Libourel, G., Goodrich, C.A., Petaev, M.I.: Silica-rich igneous rims around magnesian chondrules in CR carbonaceous chondrites: evidence for condensation origin from fractionated nebular gas. Meteorit. Planet. Sci. 39, 1931-1955 (2004). doi:10.1111/j.19455100.2004.tb00088.x

Krot, A.N., Amelin, Y., Cassen, P., Meibom, A.: Young chondrules in CB chondrites from a giant impact in the early Solar System. Nature 436, 989-992 (2005a). doi:10.1038/nature03830

Krot, A.N., Yurimoto, H., Hutcheon, I.D., MacPherson, G.J.: Chronology of the early Solar System from chondrule-bearing calcium-aluminium-rich inclusions. Nature 434, 998-1001 (2005b). doi: $10.1038 /$ nature 03470

Krot, A.N., Libourel, G., Chaussidon, M.: Oxygen isotope compositions of chondrules in CR chondrites. Geochim. Cosmochim. Acta 70, 767-779 (2006). doi:10.1016/j.gca.2005.08.028

Krot, A.N., Ivanova, M.A., Ulyanov, A.A.: Chondrules in the CB/CH-like carbonaceous chondrite isheyevo: evidence for various chondrule-forming mechanisms and multiple chondrule generations. Chem. Erde 67, 283-300 (2007). doi:10.1016/j.chemer.2006.04.001. http://www. sciencedirect.com/science/article/pii/S0009281906000213

Krot, A.N., et al.: Origin and chronology of chondritic components: a review. Geochim. Cosmochim. Acta 73, 4963 (2009)

Krot, A.N., Nagashima, K., van Kooten, E.M.M.E., Bizzarro, M.: Calcium-aluminum-rich inclusions recycled during formation of porphyritic chondrules from $\mathrm{CH}$ carbonaceous chondrites. Geochim. Cosmochim. Acta 201, 185-223 (2017). doi:10.1111/j.1945-5100.2002.tb00805.x

Kruijer, T.S., Touboul, M., Fischer-Gödde, M., Bermingham, K.R., Walker, R.J., Kleine, T.: Protracted core formation and rapid accretion of protoplanets. Science 344, 1150-1154 (2014). doi:10.1126/science. 1251766 
Kunihiro, T., Rubin, A.E., McKeegan, K.D., Wasson, J.T.: Initial ${ }^{26} \mathrm{Al} /{ }^{27} \mathrm{Al}$ in carbonaceouschondrite chondrules: too little ${ }^{26} \mathrm{Al}$ to melt asteroids. Geochim. Cosmochim. Acta 68, 2947-2957 (2004). doi:10.1016/j.gca.2004.02.006

Kurahashi, E., Kita, N.T., Nagahara, H., Morishita, Y.: ${ }^{26} \mathrm{Al}-{ }^{26} \mathrm{Mg}$ systematics of chondrules in a primitive CO chondrite. Geochim. Cosmochim. Acta 72, 3865-3882 (2008). doi:10.1016/j.gca.2008.05.038

Lambrechts, M., Johansen, A.: Rapid growth of gas-giant cores by pebble accretion. Astron. Astrophys. 544, A32 (2012). doi:10.1051/0004-6361/201219127, 1205.3030

Lambrechts, M., Johansen, A., Morbidelli, A.: Separating gas-giant and ice-giant planets by halting pebble accretion. Astron. Astrophys. 572, A35 (2014). doi:10.1051/0004-6361/201423814, 1408.6087

Larsen, K.K., Trinquier, A., Paton, C., Schiller, M., Wielandt, D., Ivanova, M.A., Connelly, J.N., Nordlund, Å., Krot, A.N., Bizzarro, M.: Evidence for magnesium isotope heterogeneity in the solar protoplanetary disk. Astrophys. J. Lett. 735, L37 (2011). doi:10.1088/20418205/735/2/L37

Larsen, K.K., Schiller, M., Bizzarro, M.: Accretion timescales and style of asteroidal differentiation in an ${ }^{26} \mathrm{Al}$-poor protoplanetary disk. Geochim. Cosmochim. Acta 176, 295-315 (2016). doi:10.1016/j.gca.2015.10.036

Libourel, G., Krot, A.N.: Evidence for the presence of planetesimal material among the precursors of magnesian chondrules of nebular origin. Earth Planet. Sci. Lett. 254, 1-8 (2007). doi:10.1016/j.epsl.2006.11.013

Luu, T.H., Young, E.D., Gounelle, M., Chaussidon, M.: Short time interval for condensation of high-temperature silicates in the solar accretion disk. Proc. Natl. Acad. Sci. 112(5), 1298-1303 (2015). doi:10.1073/pnas.1414025112. http://www.pnas.org/content/112/5/1298

MacPherson, G.J., Kita, N.T., Ushikubo, T., Bullock, E.S., Davis, A.M.: Well-resolved variations in the formation ages for Ca-Al-rich inclusions in the early Solar System. Earth Planet. Sci. Lett. 331-332, 43-54 (2012). doi:10.1016/j.epsl.2012.03.010. http://www.sciencedirect.com/ science/article/pii/S0012821X12001240

Makide, K., Nagashima, K., Krot, A.N., Huss, G.R., Hutcheon, I.D., Bischoff, A.: Oxygen- and magnesium-isotope compositions of calcium-aluminum-rich inclusions from CR2 carbonaceous chondrites. Geochim. Cosmochim. Acta 73, 5018-5050 (2009). doi:10.1016/j.gca.2009.01.042

McKeegan, K.D., Chaussidon, M., Robert, F.: Incorporation of short-lived ${ }^{10} \mathrm{Be}$ in a calciumaluminum-rich inclusion from the allende meteorite. Science 289, 1334-1337 (2000). doi:10.1126/science.289.5483.1334

McKeegan, K.D., Kallio, A.P.A., Heber, V.S., Jarzebinski, G., Mao, P.H., Coath, C.D., Kunihiro, T., Wiens, R.C., Nordholt, J.E., Moses, R.W., Reisenfeld, D.B., Jurewicz, A.J.G., Burnett, D.S.: The oxygen isotopic composition of the Sun inferred from captured solar wind. Science 332, 1528 (2011). doi:10.1126/science. 1204636

McNally, C.P., Hubbard, A., Mac Low, M.M., Ebel, D.S., D’Alessio, P.: Mineral processing by short circuits in protoplanetary disks. Astrophys. J. Lett. 767, L2 (2013). doi:10.1088/20418205/767/1/L2, 1301.1698

McNaughton, N.J., Borthwick, J., Fallick, A.E., Pillinger, C.T.: Deuterium/hydrogen ratios in unequilibrated ordinary chondrites. Nature 294, 639-641 (1981). doi:10.1038/294639a0

Morris, M.A., Boley, A.C., Desch, S.J., Athanassiadou, T.: Chondrule formation in bow shocks around eccentric planetary embryos. Astrophys. J. 752, 27 (2012). doi:10.1088/0004637X/752/1/27, 1204.0739

Müller, T.W.A., Kley, W.: Modelling accretion in transitional disks. Astron. Astrophys. 560, A40 (2013). doi:10.1051/0004-6361/201322503, 1310.4398

Nagashima, K., Krot, A.N., Huss, G.R.: Oxygen-isotope compositions of chondrule phenocrysts and matrix grains in Kakangari K-grouplet chondrite: implication to a chondrule-matrix genetic relationship. Geochim. Cosmochim. Acta 151, 49-67 (2015). doi:10.1016/j.gca.2014.12.012 
Nesvorný, D., Jenniskens, P., Levison, H.F., Bottke, W.F., Vokrouhlický, D., Gounelle, M.: Cometary origin of the zodiacal cloud and carbonaceous micrometeorites. Implications for hot debris disks. Astrophys. J. 713, 816-836 (2010). doi:10.1088/0004-637X/713/2/816, 0909. 4322

Norris, T.L., Gancarz, A.J., Rokop, D.J., Thomas, K.W.: Half-life of ${ }^{26}$ Al. J. Geophys. Res. 88, B331-B333 (1983). doi:10.1029/JB088iS01p0B331

Olsen, M.B., Schiller, M., Krot, A.N., Bizzarro, M.: Magnesium isotope evidence for single stage formation of $\mathrm{CB}$ chondrules by colliding planetesimals. Astrophys. J. Lett. 776, L1 (2013). doi:10.1088/2041-8205/776/1/L1

Olsen, M.B., Wielandt, D., Schiller, M., Van Kooten, E.M.M.E., Bizzarro, M.: Magnesium and ${ }^{54} \mathrm{Cr}$ isotope compositions of carbonaceous chondrite chondrules - Insights into early disk processes. Geochim. Cosmochim. Acta 191, 118-138 (2016). doi:10.1016/j.gca.2016.07.011

Oulton, J., Humayun, M., Fedkin, A., Grossman, L.: Chemical evidence for differentiation, evaporation and recondensation from silicate clasts in Gujba. Geochim. Cosmochim. Acta 177, 254-274 (2016). doi:10.1016/j.gca.2016.01.008

Palme, H., Hezel, D.C., Ebel, D.S.: The origin of chondrules: constraints from matrix composition and matrix-chondrule complementarity. Earth Planet. Sci. Lett. 411, 11-19 (2015). doi:10.1016/j.epsl.2014.11.033

Paton, C., Schiller, M., Bizzarro, M.: Identification of an ${ }^{84}$ Sr-depleted carrier in primitive meteorites and implications for thermal processing in the solar protoplanetary disk. Astrophys. J. Lett. 763, L40 (2013). doi:10.1088/2041-8205/763/2/L40

Qin, L., Alexander, C.M.O., Carlson, R.W., Horan, M.F., Yokoyama, T.: Contributors to chromium isotope variation of meteorites. Geochim. Cosmochim. Acta 74, 1122-1145 (2010). doi:10.1016/j.gca.2009.11.005

Regelous, M., Elliott, T., Coath, C.D.: Nickel isotope heterogeneity in the early Solar System. Earth Planet. Sci. Lett. 272, 330-338 (2008). doi:10.1016/j.eps1.2008.05.001

Reipurth, B., Bally, J.: Herbig-Haro flows: probes of early stellar evolution. Annu. Rev. Astron. Astrophys. 39, 403-455 (2001). doi:10.1146/annurev.astro.39.1.403

Robert, F., Epstein, S.: The concentration and isotopic composition of hydrogen, carbon and nitrogen in carbonaceous meteorites. Geochim. Cosmochim. Acta 46, 81-95 (1982). doi:10.1016/0016-7037(82)90293-9

Robert, F., Javoy, M., Halbout, J., Dimon, B., Merlivat, L.: Hydrogen isotope abundances in the solar system. I - Unequilibrated chondrites. II - Meteorites with terrestrial-like D/H ratio. Geochim. Cosmochim. Acta 51, 1787-1805 (1987). doi:10.1016/0016-7037(87)90170-0

Romanova, M.M., Ustyugova, G.V., Koldoba, A.V., Lovelace, R.V.E.: Launching of conical winds and axial jets from the disc-magnetosphere boundary: axisymmetric and 3D simulations. Mon. Not R. Astron. Soc. 399, 1802-1828 (2009). doi:10.1111/j.1365-2966.2009.15413.x, 0907. 3394

Rudraswami, N.G., Ushikubo, T., Nakashima, D., Kita, N.T.: Oxygen isotope systematics of chondrules in the Allende CV3 chondrite: high precision ion microprobe studies. Geochim. Cosmochim. Acta 75, 7596-7611 (2011). doi:10.1016/j.gca.2011.09.035

Russell, S.S., Krot, A.N., Huss, G.R., Keil, K., Itoh, S., Yurimoto, H., MacPherson, G.J.: The genetic relationship between refractory inclusions and chondrules. In: Krot, A.N., Scott, E.R.D., Reipurth, B. (eds.) Chondrites and the Protoplanetary Disk. Astronomical Society of the Pacific Conference Series, vol. 341, p. 317. Astronomical Society of the Pacific, San Francisco (2005)

Salmeron, R., Ireland, T.R.: Formation of chondrules in magnetic winds blowing through the protoasteroid belt. Earth Planet. Sci. Lett. 327, 61-67 (2012). doi:10.1016/j.eps1.2012.01.033

Sanders, I.S., Scott, E.R.D.L.: The origin of chondrules and chondrites: debris from lowvelocity impacts between molten planetesimals? Meteorit. Planet. Sci. 47, 2170-2192 (2012). doi:10.1111/maps.12002

Schiller, M., et al.: Rapid timescales for magma ocean crystallization on the howardite-eucritediogenite parent body. Astrophys. J. Lett. 740, L22 (2011). doi:10.1088/2041-8205/740/1/L22 
Schiller, M., Connelly, J.N., Glad, A.C., Mikouchi, T., Bizzarro, M.: Early accretion of protoplanets inferred from a reduced inner solar system ${ }^{26} \mathrm{Al}$ inventory. Earth Planet. Sci. Lett. 420, 45-54 (2015a). doi:10.1016/j.epsl.2015.03.028

Schiller, M., Paton, C., Bizzarro, M.: Evidence for nucleosynthetic enrichment of the protosolar molecular cloud core by multiple supernova events. Geochim. Cosmochim. Acta 149, 88102 (2015b). doi:10.1016/j.gca.2014.11.005. http://www.sciencedirect.com/science/article/pii/ S001670371400667X

Schrader, D.L., Nagashima, K., Krot, A.N., Ogliore, R.C., Hellebrand, E.: Variations in the O-isotope composition of gas during the formation of chondrules from the CR chondrites. Geochim. Cosmochim. Acta 132, 50-74 (2014). doi:10.1016/j.gca.2014.01.034

Scott, E.R.D.: Chondrites and the protoplanetary disk. Annu. Rev. Earth Plan. Sci. 35, 577 (2007). doi:10.1146/annurev.earth.35.031306.140100. http://adsabs.harvard.edu/abs/2007AREPS..35. $577 \mathrm{~S}$

Scott, E.R.D., Krot, A.N.: Chondrites and Their Components, p. 143. Elsevier, Amsterdam (2005)

Sheikhnezami, S., Fendt, C., Porth, O., Vaidya, B., Ghanbari, J.: Bipolar jets launched from magnetically diffusive accretion disks. I. ejection efficiency versus field strength and diffusivity. Astrophys. J. 757, 65 (2012). doi:10.1088/0004-637X/757/1/65, 1207.6086

Shu, F.H., Shang, H., Lee, T.: Toward an astrophysical theory of chondrites. Science 271, 15451552 (1996). doi:10.1126/science.271.5255.1545

Shu, F.H., Shang, H., Glassgold, A.E., Lee, T.: X-rays and fluctuating X-winds from protostars. Science 277, 1475-1479 (1997). doi:10.1126/science.277.5331.1475

Soulié, C., Libourel, G., Tissandier, L., Hiver, J.M.: Kinetics of olivine dissolution in chondrule melts: an experimental study. In: Lunar and Planetary Science Conference, vol 43, p. 1840 (2012)

Tenner, T.J., Nakashima, D., Ushikubo, T., Kita, N.T., Weisberg, M.K.: Oxygen isotope ratios of $\mathrm{FeO}$-poor chondrules in CR3 chondrites: influence of dust enrichment and $\mathrm{H}_{2} \mathrm{O}$ during chondrule formation. Geochim. Cosmochim. Acta 148, 228-250 (2015). doi:10.1016/j.gca.2014.09.025

Tissot, F.L.H., Dauphas, N., Grossman, L.: Origin of uranium isotope variations in early solar nebula condensates. Sci. Adv. 2, e1501400-e1501400 (2016). doi:10.1126/sciadv.1501400, 1603.01780

Trinquier, A., Birck, J.L., Allègre, C.J.: Widespread ${ }^{54} \mathrm{Cr}$ Heterogeneity in the Inner Solar System. Astrophys. J. 655, 1179-1185 (2007). doi:10.1086/510360

Trinquier, A., Elliott, T., Ulfbeck, D., Coath, C., Krot, A.N., Bizzarro, M.: Origin of nucleosynthetic isotope heterogeneity in the solar protoplanetary disk. Science 324, 374 (2009). doi: $10.1126 /$ science. 1168221

Ushikubo, T., Nakashima, D., Kimura, M., Tenner, T.J., Kita, N.T.: Contemporaneous formation of chondrules in distinct oxygen isotope reservoirs. Geochim. Cosmochim. Acta 109, 280-295 (2013). doi:10.1016/j.gca.2013.01.045

Van Kooten, E.M.M.E., Wielandt, D., Schiller, M., Nagashima, K., Thomen, A., Larsen, K.K., Olsen, M.B., Nordlund, A., Krot, A.N., Bizzarro, M.: Isotopic evidence for primordial molecular cloud material in metal-rich carbonaceous chondrites. Proc. Natl. Acad. Sci. USA 113(8), 2011-2016 (2016). doi:10.1073/pnas.1518183113. http://www.pnas.org/content/113/8/ 2011

Villeneuve, J., Chaussidon, M., Libourel, G.: Homogeneous distribution of ${ }^{26} \mathrm{Al}$ in the Solar System from the Mg isotopic composition of chondrules. Science 325(5943), 985-988 (2009). doi:10.1126/science.1173907. http://www.sciencemag.org/cgi/content/abstract/325/5943/985

Walsh, K.J., Morbidelli, A., Raymond, S.N., O'Brien, D.P., Mandell, A.M.: A low mass for Mars from Jupiter's early gas-driven migration. Nature 475, 206-209 (2011). doi:10.1038/nature10201, 1201.5177 
Warren, P.H.: Stable-isotopic anomalies and the accretionary assemblage of the Earth and Mars: a subordinate role for carbonaceous chondrites. Earth Planet. Sci. Lett. 311, 93-100 (2011). doi:10.1016/j.eps1.2011.08.047

Wasserburg, G.J., Busso, M., Gallino, R., Nollett, K.M.: Short-lived nuclei in the early solar system: possible AGB sources. Nucl. Phys. A 777, 5-69 (2006). doi:10.1016/j.nuclphysa.2005.07.015, astro-ph/0602551

Weidenschilling, S.J.: Aerodynamics of solid bodies in the solar nebula. Mon. Not. R. Astron. Soc. 180, 57-70 (1977). doi:10.1093/mnras/180.1.57

Weisberg, M.K., Prinz, M., Clayton, R.N., Mayeda, T.K., Grady, M.M., Pillinger, C.T.: The CR chondrite clan. Antarct. Meteorite Res. 8, 11 (1995)

Weiss, B.P., Elkins-Tanton, L.T.: Differentiated planetesimals and the parent bodies of chondrites. Annu. Rev. Earth Plan. Sci. 41, 529-560 (2013). doi:10.1146/annurev-earth-040610-133520

Williams, J.P., Cieza, L.A.: Protoplanetary disks and their evolution. Annu. Rev. Astron. Astrophys. 49, 67 (2011). doi:10.1146/annurev-astro-081710-102548;

Yamakawa, A., Yamashita, K., Makishima, A., Nakamura, E.: Chromium isotope systematics of achondrites: chronology and isotopic heterogeneity of the inner solar system bodies. Astrophys. J. 720, 150-154 (2010). doi:10.1088/0004-637X/720/1/150

Open Access This chapter is licensed under the terms of the Creative Commons Attribution 4.0 International License (http://creativecommons.org/licenses/by/4.0/), which permits use, sharing, adaptation, distribution and reproduction in any medium or format, as long as you give appropriate credit to the original author(s) and the source, provide a link to the Creative Commons license and indicate if changes were made.

The images or other third party material in this chapter are included in the chapter's Creative Commons license, unless indicated otherwise in a credit line to the material. If material is not included in the chapter's Creative Commons license and your intended use is not permitted by statutory regulation or exceeds the permitted use, you will need to obtain permission directly from the copyright holder.

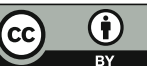

